\title{
Boundary Topological Entanglement Entropy in Two and Three Dimensions
}

\author{
Jacob C. Bridgeman ${ }^{1}$ (D), Benjamin J. Brown ${ }^{2}$ (D), Samuel J. Elman ${ }^{2,3,4}$ \\ ${ }^{1}$ Perimeter Institute for Theoretical Physics, Waterloo, ON, Canada \\ E-mail: jcbridgeman1@gmail.com; URL: https://jcbridgeman.bitbucket.io \\ 2 Centre for Engineered Quantum Systems, School of Physics, The University of Sydney, Sydney, \\ NSW 2006, Australia \\ 3 Department of Physics, Imperial College London, London SW7 2AZ, UK \\ 4 School of Physics and Astronomy, University of Leeds, Leeds LS2 9JT, UK
}

Received: 23 July 2021 / Accepted: 5 August 2021

Published online: 26 August 2021 - (C) The Author(s) 2021

\begin{abstract}
The topological entanglement entropy is used to measure long-range quantum correlations in the ground space of topological phases. Here we obtain closed form expressions for the topological entropy of $(2+1)$ - and $(3+1)$-dimensional loop gas models, both in the bulk and at their boundaries, in terms of the data of their input fusion categories and algebra objects. Central to the formulation of our results are generalized $\mathcal{S}$-matrices. We conjecture a general property of these $\mathcal{S}$-matrices, with proofs provided in many special cases. This includes constructive proofs for categories up to rank 5.
\end{abstract}

\section{Introduction}

The classification of topological phases is fundamental to the study of modern condensed matter physics [1-4]. Moreover, they have properties that may be valuable for the robust storage and manipulation of quantum information [5,6]. Their characteristics include a stable gap at zero temperature and quasiparticle excitations with non-trivial braid statistics [7,8].

An important class of topological phases are represented by topological loop-gas models $[9,10]$. These models can be defined in terms of an input unitary fusion category, and their ground states by superpositions of string diagrams labeled by objects from the category. The categorical framework provides a collection of local relations that ensure topological invariance of the ground states. In $(2+1)$-dimensions, these models are called Levin-Wen models [9]. Levin-Wen models have point-like excitations, commonly called anyons, with non-trivial fusion rules and braid statistics. In (3+1)-dimensions, the input category must be equipped with a premodular braiding, leading to a Walker-Wang model [10]. Generically, Walker-Wang models support point-like and loop-like excitations. In contrast to Levin-Wen models, the excitations in the bulk of a Walker-Wang model may be trivial, specifically if the input category is modular.

Loop-gas models can be defined on manifolds with boundaries by modifying the local relations governing the strings in the vicinity of the boundary. One way to define a 
boundary to a topological loop-gas is to allow some strings to terminate on the boundary. This is captured in the current work using particular objects called algebras $[11,12]$. Despite their trivial bulk excitations, Walker-Wang models may have highly non-trivial boundary excitations.

Intimately connected to the topological properties of these phases is the long-range entanglement present in the ground state of the Hamiltonians describing these phases $[13,14]$. The long-range quantum correlations found in the ground states of topological phases can be measured using the topological entanglement entropy [15-17]. We typically expect that the entanglement entropy shared between two subsystems of the ground state of a gapped many body system to respect an area law [18]. However, supposing a sensible choice of bipartition, the entanglement entropy of the ground state of topological phases has a constant universal correction [15]. In (2+1)-dimensions, it is known that this correction relates to the total quantum dimension of the quasiparticle excitations supported by the phase $[16,17]$. We can also evaluate the quantum dimensions of individual excitations [19] and defects [20,21] of a phase using topological entanglement entropy. Other work has shown we can use the topological entanglement entropy to calculate the fusion rules [22] and braid statistics [23] of (2+1)-dimensional phases.

Generalizations of topological entanglement entropy diagnostics have been found [24,25] for $(3+1)$-dimensional phases with bulk topological order. These diagnostics were first demonstrated using the (3+1)-dimensional toric code model [26] as an example. This phase gives rise to one species of bosonic excitation that braids non-trivially with a loop-like excitation in the bulk of the system. In contrast, particular classes of WalkerWang models [10] have been shown to behave differently using the same diagnostics. Modular examples of these models demonstrate zero bulk topological entanglement entropy [27,28], even though, at their boundary, they realize quasiparticle excitations with non-trivial braid statistics [27].

In Ref. [29], two new diagnostics were found to interrogate the long-range entanglement at the boundary of a (3+1)-dimensional-dimensional topological phase. The behavior of the diagnostics was determined by making quite generic considerations of the support of creation operators for topological excitations, without assuming any knowledge of the underlying particle theory of the phase. It was shown that the diagnostics will show a null outcome only if all the particles that can be created at the boundary have trivial braid statistics. Conversely, boundary topological order must necessarily show positive topological entanglement entropy if quasi-particles that demonstrate nontrivial braid statistics can be created. In that work, the diagnostics were tested at the different boundaries of the $(3+1)$-dimensional-dimensional toric code where null outcomes were obtained at boundaries where the appropriate types of particles condense. However, a limitation of the diagnostics presented in that paper is that the meaning of a positive outcome is not well understood.

From the input fusion category perspective, the topological entanglement entropies can be understood as arising from constraints on the 'string flux' passing through a surface. In (3+1)-dimensions, there are also additional corrections due to braiding. Allowing strings to terminate in the vicinity of a physical boundary alters the flux (and braiding) constraints in the vicinity, thereby altering the topological entropy.

In this work, we obtain closed form expressions for bulk and boundary topological entanglement entropy diagnostics for topological loop-gas models. We obtain our results by evaluating the entanglement entropy of various regions of ground states of Levin-Wen and Walker-Wang models. This requires careful analysis of various string diagrams, such 
Table 1. Summary of results, technical terms defined in Sect. 2. The bulk strings are labeled by a unitary fusion category $\mathcal{C}$, possibly with extra structure. The number $\mathcal{D}$ denotes the total quantum dimension of $\mathcal{C}, A$ is a Q-system (with extra structure, see Sect. 2) of dimension $d_{A}, \mathcal{Z}(\mathcal{C})$ and $\mathcal{Z}_{2}(\mathcal{C})$ are the Drinfeld and Müger centers of $\mathcal{C}$ respectively. Topological entanglement entropies for Levin-Wen models are denoted $\gamma$ and $\Gamma$ for the bulk and near the boundary respectively. These quantities are defined in Eq. (10), Eq. (11) and Fig. 2. The corresponding quantities in (3+1)-dimensions are denoted $\delta$ for the bulk entropy (Eq. (12) and Fig. 3), and $\Delta_{\bullet}\left(\Delta_{\circ}\right)$ for the boundary entropy detecting point-like (loop-like) excitations as defined in Eq. (13) and Fig. 4 (Eq. (14) and Fig. 5)

\begin{tabular}{|c|c|c|c|}
\hline Model & Bulk strings & Boundary Q-system & Topological entropy \\
\hline \multirow[t]{2}{*}{ Levin-Wen } & $\mathcal{C}$ fusion & & $\gamma=\log \mathcal{D}_{\mathcal{Z}(\mathcal{C})}^{2}$ \\
\hline & & $A$ & $\Gamma=\log \mathcal{D}^{2}$ \\
\hline \multirow[t]{3}{*}{ Walker-Wang } & $\mathcal{C}$ premodular & & $\delta=\mathcal{D}_{\mathcal{Z}_{2}(\mathcal{C})}^{2}{ }^{a}$ \\
\hline & & $A$ & $\begin{array}{l}\Delta_{\bullet}=?^{\mathrm{b}} \\
\Delta_{\circ}=\log d_{A}^{2}-\log \mathcal{D}^{2}+\Delta_{\bullet}\end{array}$ \\
\hline & & $A=1$ & $\begin{array}{l}\Delta_{\bullet}=\log \mathcal{D}^{2} \\
\Delta_{\circ}=0\end{array}$ \\
\hline \multirow[t]{2}{*}{ Walker-Wang } & $\mathcal{C}$ symmetric & & $\delta=\log \mathcal{D}_{\mathcal{Z}_{2}(\mathcal{C})}^{2}$ \\
\hline & & $A$ & $\begin{array}{l}\Delta_{\bullet}=\log \mathcal{D}^{2}-\log d_{A} \\
\Delta_{\circ}=\log d_{A}\end{array}$ \\
\hline \multirow[t]{3}{*}{ Walker-Wang } & $\mathcal{C}$ pointed & & $\delta=\log \mathcal{D}_{\mathcal{Z}_{2}(\mathcal{C})}^{2}$ \\
\hline & & $A$ such that $A \cap \mathcal{Z}_{2}(\mathcal{C})=\{1\}^{\mathrm{c}}$ & $\begin{array}{l}\Delta_{\bullet}=\log \mathcal{D}^{2}-2 \log d_{A} \\
\Delta_{\circ}=0\end{array}$ \\
\hline & & $A$ such that $A \cap \mathcal{Z}_{2}(\mathcal{C})=A$ & $\begin{array}{l}\Delta_{\bullet}=\log \mathcal{D}^{2}-\log d_{A} \\
\Delta_{\circ}=\log d_{A}\end{array}$ \\
\hline
\end{tabular}

${ }^{a}$ Conjectured, proven in many cases as indicated in Theorem 3.

b We do not have a general form at present.

c Includes the case $\mathcal{C}$ modular

as generalized $\mathcal{S}$-matrices which encode the braiding properties of the input category. Additionally, we examine how the inclusion of boundaries, via algebra objects, alter these diagrams, and so the topological entropy. In all cases, we find that the entropy can be expressed in terms of the quantum dimension of the input category and the quantum dimension of the algebra object. In the bulk of (3+1)-dimensional models, we conjecture, and prove in many cases, that the entropy is the logarithm of the total quantum dimension of the particle content of the theory, extending the results of Ref. [28].

Overview. Following a brief summary of our results, the remainder of this paper is structured as follows. In Sect. 2, we introduce some notation and minor results that are required for the remainder of the paper. In Sect. 3, we briefly review the models of interest, and discuss the class of boundaries we consider. In Sect. 4, we explain the origin and meaning of topological entanglement entropy, and define the diagnostics used to detect boundary topological entanglement entropy. In Sect. 5, we compute the entropy of bulk regions for Levin-Wen models. These computations are required for the Walker-Wang models, and provide a good warm-up. We then discuss the additional considerations for Walker-Wang models, and extend the computations to these cases. In Sect. 6, we compute the boundary entropy diagnostics for Levin-Wen models with boundary, followed by some classes of Walker-Wang models with boundary. We summarize in Sect. 7.

We include two appendices. In Appendix A, we provide proofs of some results concerning generalized $\mathcal{S}$-matrices. In Appendix B, we provide proofs of some results concerning loop-gas models, and their entropies. 
1.1. Summary of results. In Table 1, we summarize our main results. The models we discuss will be introduced in the following sections, followed by the proofs of these results. We note that many of these results were previously known, for example the bulk Levin-Wen appears in Refs. [16,17]. When the Levin-Wen model is defined by the fusion category $\operatorname{Vec}(G)$, the boundary Levin-Wen results appear in Ref. [30]. The bulk results for symmetric and modular Walker-Wang models appear in Ref. [28]. We extend this to include all pointed inputs (all quantum dimensions equal to 1), as well as all input categories up to rank 5. This allows us to conjecture a general result. To the best of our knowledge, there are no results concerning boundary entropies of Walker-Wang models beyond the (3+1)-dimensional toric code [29].

\section{Preliminaries}

In this work, each of the (2+1)-dimensional models we are interested in are described by a unitary fusion category. In $(3+1)$ dimensions we use a unitary premodular category. Boundaries of these models can be specified using algebra objects in the input category. For definitions of the various algebraic objects, we refer to Ref. [31], or for the physically minded reader Refs. [32-34]. Here, we briefly review the notation we use to describe these objects.

Let $\mathcal{C}$ be a fusion category (Ref. [31], Def. 4.1.1). Without loss of generality, we assume $\mathcal{C}$ is skeletal (isomorphic objects are equal), and the unit is strict. The number of simple objects is called the rank of $\mathcal{C}$, denoted $\operatorname{rk}(\mathcal{C})$. In all cases, we denote the unit object of $\mathcal{C}$ by 1 , and the dual of an object $x$ by $\bar{x}$.

The category $\mathcal{C}$ is equipped with a bilinear functor $\otimes: \mathcal{C} \times \mathcal{C} \rightarrow \mathcal{C}$. Once we fix a basis for the fusion space $\mathcal{C}(a \otimes b, c)$, we denote a basis vector $\mu$ using a trivalent vertex [35]

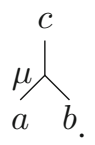

The (vector space) dimension of $\mathcal{C}(a \otimes b, c)$ is denoted $N_{a, b}^{c}$. We call these tensors the fusion rules of $\mathcal{C}$. If all $N_{a, b}^{c} \in\{0,1\}$, we call the category multiplicity free. We normalize these bases following Ref. [33].

The natural isomorphism $(-\otimes-) \otimes-\cong-\otimes(-\otimes-)$ is realized as the $F$-matrices [32] of $\mathcal{C}$, and we refer to such a re-association as an $F$-move. If these are unitary, we say that $\mathcal{C}$ is a unitary fusion category. Henceforth, we restrict to the unitary setting. We assume that $\mathcal{C}$ is equipped with the unique unitary spherical structure [36], meaning that all diagrams can be treated as though they are drawn on the surface of a sphere.

The dimension of a simple object $a$ is denoted $d_{a}$, while the total dimension of $\mathcal{C}$ is denoted $\mathcal{D}_{\mathcal{C}}^{2}:=\sum_{a} d_{a}^{2}$, where the sum is over all simple objects. When it is clear from context, we omit the subscript. In string diagrams, a loop labeled by $a$ is assigned $d_{a}$. We refer to insertion or removal of a loop, and the associated division or multiplication by the dimension, as a loop move. If $\mathcal{D}^{2}=\operatorname{rk}(\mathcal{C})$, we say that $\mathcal{C}$ is pointed. This property is also commonly called Abelian in the physics literature.

Braided unitary fusion categories (Ref. [31], Definition 8.1.2) can be uniquely equipped with a unitary premodular structure [37]. For the results in this manuscript, it is important to understand which strings can be 'uncrossed'. This is captured by the Müger 
center (Ref. [38], Definition 2.9), denoted $\mathcal{Z}_{2}(\mathcal{C})$. A premodular category is symmetric if $\mathcal{Z}_{2}(\mathcal{C})=\mathcal{C}$, and modular if $\mathcal{Z}_{2}(\mathcal{C})=$ Vec. We will refer to premodular $\overline{\text { categories }}$ which are neither symmetric nor modular as properly premodular.

We will make extensive use of an operator we call the connected $\mathcal{S}$-matrix $\mathcal{S}_{c}$, with matrix elements

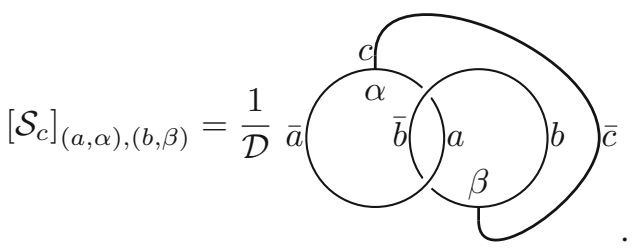

The usual $\mathcal{S}$-matrix (Ref. [38], Definition 2.2) occurs as a special case, namely $\mathcal{S}_{a, b}=$ $\left[\mathcal{S}_{1}\right]_{a, b}$. The connected $\mathcal{S}$-matrix appears in Theorem 3.1.17 of Ref. [39], and is closely related to the punctured $\mathcal{S}$-matrix of Ref. [40]. We will need the following result concerning the connected $\mathcal{S}$-matrix.

Lemma 1. Let $\mathcal{C}$ be a unitary premodular category, then

$$
\sum_{c \in \mathcal{C}} \operatorname{Tr} \mathcal{S}_{c}^{\dagger} \mathcal{S}_{c}=\mathcal{D}^{2}
$$

where $\mathcal{S}_{c}$ is the connected $\mathcal{S}$-matrix and $\mathcal{D}$ is the total dimension of $\mathcal{C}$.

Proof. Provided in Appendix A.

We now briefly review the notation we use to describe algebra objects in $\mathcal{C}$. Let $(A, m, \eta)$ be a Q-system, also called a unitary Frobenius algebra, in $\mathcal{C}$ (Ref. [41], Def. 5.4). Since $\mathcal{C}$ is semi-simple, we can decompose the object $A=1 \oplus a_{1} \oplus a_{2} \oplus \ldots$.. In this work, we restrict to the multiplicity free case, so each $a_{i}$ appears at most once. We represent the multiplication morphism $m$ as

$$
m=\bigwedge_{A A}^{A}=\sum_{a, b, c \in A} m_{a, b}^{c} \bigwedge_{a}^{c} .
$$

To simplify the notation, we define $m_{x, y}^{z}=0$ whenever any of the labels do not occur in the decomposition of $A$. This allows us to always sum over simple objects in $\mathcal{C}$. Additionally, we suppress the $A$ label. Any unlabeled lines carry an implicit $A$. We can always normalize the unit morphism $\eta=1$, and the multiplication morphism

$$
\sum_{a, b \in A}\left|m_{a, b}^{c}\right|^{2} \sqrt{d_{a} d_{b}}=\delta_{c \in A} \sqrt{d_{c}} d_{A},
$$

where $d_{A}=\sum_{a \in A} d_{a}$. When it does not cause confusion, we will indicate a Q-system by its object, for example $A=1$, the 'unit algebra'.

If the underlying category $\mathcal{C}$ is braided, we say the algebra is commutative if it commutes in the category (Ref. [31], Definition 8.8.1). 
2.1. Examples. We use the following examples throughout our work to illustrate our results. The unitary fusion category $\operatorname{Vec}^{\omega}\left(\mathbb{Z}_{2}\right)$ is the category of finite dimensional $\mathbb{Z}_{2-}$ graded vector spaces. The simple objects of this pointed category are group elements $\mathbb{Z}_{2}:=\left\{1, x \mid x^{2}=1\right\}$. We neglect to draw the unit object, 1 . As such, the only nonzero trivalent vertex is

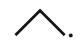

There are exactly two inequivalent associators compatible with the fusion rule, namely

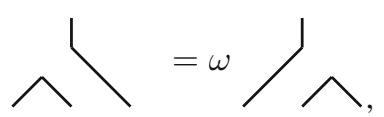

with $\omega= \pm 1$. With the associator fixed, there are two compatible braidings

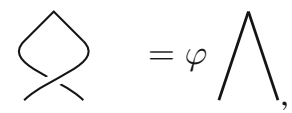

with $\varphi^{2}=\omega$. We denote by $\operatorname{Vec}^{\omega, \varphi}\left(\mathbb{Z}_{2}\right)$ the braided category, with associator $\omega$ and braiding $\varphi$. The categories $\operatorname{Vec}^{-1, \pm i}\left(\mathbb{Z}_{2}\right)$ are modular, while Vec ${ }^{1, \pm 1}\left(\mathbb{Z}_{2}\right)$ are symmetric.

These four examples are included in the attached Mathematica file [42] as $\operatorname{Vec}^{1,1}\left(\mathbb{Z}_{2}\right)=\mathrm{FR}_{1 ; 0}^{2,0}, \operatorname{Vec}^{1,-1}\left(\mathbb{Z}_{2}\right)=\mathrm{FR}_{1 ; 2}^{2,0}, \operatorname{Vec}^{-1, i}\left(\mathbb{Z}_{2}\right)=\mathrm{FR}_{1 ; 1}^{2,0}$, and $\operatorname{Vec}^{-1,-i}\left(\mathbb{Z}_{2}\right)=$ $\mathrm{FR}_{1 ; 3}^{2,0}$.

For these examples, there are two possible Q-systems, namely $A_{0}:=1$, with trivial $m$ morphism, and $A_{1}:=1 \oplus x$. The unit algebra, $A_{0}$, is compatible as a commutative Q-system. For $A_{1}$, compatibility as a Q-system reduces to $m_{x, x}^{1} \omega=m_{x, x}^{1}$, so is only a valid algebra object when $\omega=1$. In that case, $A_{1}$ is commutative when $m_{x, x}^{1} \varphi=m_{x, x}^{1}$, which required $\varphi=1$.

\section{Loop-gas Models in (2+1) and (3+1) Dimensions}

In this work, we study loop-gas models. In their most general form, these models have ground states described by superpositions of string diagrams subject to a collection of rules, for example a diagram may be declared invalid in a ground state superposition if it contains an 'open string'. We focus on topological loop-gas models. In this case, the rules are a collection of local manipulations or moves under which states must be invariant. These are designed to ensure invariance under diffeomorphism. Given a triangulation of the manifold, which provides a lattice structure on which a condensed matter model can be defined [43], the local moves ensure retriangulation invariance.

Levin-Wen [4,9] and Walker-Wang [10,44-46] models are, respectively, two- and three-dimensional Hamiltonian models that give rise to topological loop-gas states as their ground states. Hamiltonians for these models are given in Ref. [9] and Ref. [10] for Levin-Wen and Walker-Wang models respectively. Our results do not depend on the particular form of the Hamiltonian, rather on universal properties of the ground states in the associated topological phase.

Quasiparticle excitations in these models are defects in the ground state, corresponding to a local change in the rules. Far from the excitations, the excited state remains invariant under the original moves but, for example, a string may be allowed to terminate at the location of the excitation. 
3.1. Bulk. We now briefly introduce the categorical description of the models of interest far from any physical boundary. We begin by discussing the bulk for the the $(2+1)$ dimensional case, namely, the Levin-Wen models before proceeding to discuss the bulk of the (3+1)-dimensional Walker Wang models.

Levin-Wen models Given a unitary fusion category $\mathcal{C}$, and a given lattice embedded on a two-dimensional manifold, the ground states of Levin-Wen models are superpositions of closed diagrams from the category. Strings lie along the edges of the lattice, and closed means they cannot terminate. To produce a lattice model, $\operatorname{rk}(\mathcal{C})$-dimensional vector spaces are assigned to each edge. These vector spaces are equipped with an orthogonal basis consisting of the objects of $\mathcal{C}$. If the category is multiplicity free, vector spaces corresponding to the fusion spaces are assigned to the vertices [47]. An abstract string configuration is realized by the vector consisting of the appropriate basis vector on each edge. At the vertices, the fusion rules of the category dictate which strings can fuse. If a given configuration occurs in a particular ground state, then any other configuration that is obtainable by local moves (i.e. $F$ - or loop-moves) also occurs in that ground state. The relative coefficients are dictated by the $F$-symbols, and consistency is ensured by the pentagon equation. Since the allowed moves are all local, there may be multiple ground states on manifolds with nontrivial genus. For example, a loop enclosing a cycle of the torus cannot be removed with the local loop move.

The collection of excitations (anyons) resulting from the Levin-Wen construction is called the Drinfeld center, denoted $\mathcal{Z}(\mathcal{C})$. We refer to Ref. [31] for a formal definition.

Walker-Wang models In (3+1)-dimensions, for Walker-Wang models, the diagrams can also include crossings. This required additional data to be added to the category, in particular a braiding. If we picture these diagrams embedded in 3 dimensional space, there is an ambiguity involved in these crossing. For example, if we look at a crossing from 'the side', there is no crossing. This ambiguity can be resolved by widening the strings into ribbons. This is implemented by insisting that the braided category is premodular.

Given a premodular category $\mathcal{C}$, and a lattice embedded in a 3-dimensional manifold, a Walker-Wang model is defined in essentially the same way as a Levin-Wen model. In addition to the $F$ - and loop- moves, $R$ - moves and insertion of links (or knots), such as the $\mathcal{S}$-matrix are allowed. Again, given any closed string configuration, any other configuration that can be reached via these rules is included in the ground state superposition.

Within the collection of string types, the subset that can be 'unlinked' from any other string is called the Müger center of $\mathcal{C}$, denoted $\mathcal{Z}_{2}(\mathcal{C})$. The Müger center labels the particle excitations of the Walker-Wang model [48].

3.2. Boundaries. To include a physical boundary in a loop-gas model, the rules must be modified. For the topological loop-gas models, these rules are again defined by local moves in the vicinity of the boundary. These must be compatible with the bulk moves, and ensure topological/retriangulation invariance at the boundary. We restrict our attention to gapped boundaries.

Levin-Wen models There are various equivalent classifications for the gapped boundaries of Levin-Wen models [11,12,49-52]. In this work, we use an internal classification. In this framework, gapped boundary conditions for Levin-Wen models are labeled by 


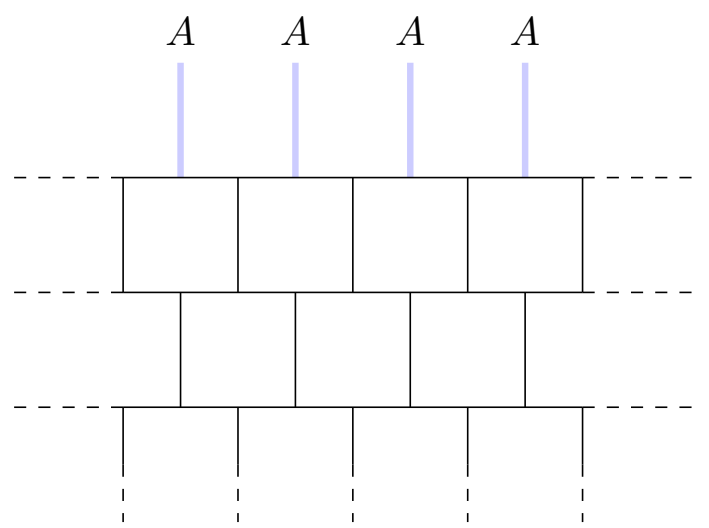

Fig. 1. An algebra specifies a boundary for a Levin-Wen model on a 'comb lattice'. Dashed lines indicate the lattice continues. The top, thick blue lines are labeled by an algebra $A$ that defines a physical boundary to the lattice

indecomposable Q-systems in $\mathcal{C}$ up to Morita equivalence $[11,12]$. We restrict to multiplicity free algebras for simplicity. These algebra objects are (not necessarily simple) objects in $\mathcal{C}$, and their simple subjects are roughly the string types that are allowed to terminate on the boundary.

On the comb lattice (Fig. 1), for example, the dangling edges only take values in the chosen algebra. Far from the boundary, the ground states look just like those with no boundary. Near the boundary, loops are no longer required to be closed, rather they can terminate on the boundary if their label occurs within the algebra. We refer to Refs. $[11,12]$ for more details, including an explicit Hamiltonian.

Walker-Wang models Just as in the bulk, when me move to (3+1)-dimensions, the braiding must be taken into account. A general classification of gapped boundaries for WalkerWang models has not been established, so we proceed for a class of boundaries generalizing those for Levin-Wen introduced above. As before, a boundary is labeled by an algebra object. Since the bulk is braided, an additional compatibility condition is required, namely that the algebra is commutative (Ref. [31], Definition 8.8.1). Finally, in this work, an indecomposable, commutative, Q-systems labels a gapped boundary condition of a Walker-Wang model [53].

3.3. Examples. Recall the examples from Sect. 2.1. In $(2+1)$-dimensions, $\operatorname{Vec}^{1, \pm 1}\left(\mathbb{Z}_{2}\right)$ lead to the same loop-gas model, since the Levin-Wen construction doesn't make use of the braiding. This model is the equally weighted superposition of all loop diagrams (with no branching due to the fusion rules). This is the ground state of the toric code model [5].

Likewise, $\operatorname{Vec}^{ \pm i}(-1)$ correspond to the same loop-gas model. Due to the nontrivial Frobenius-Schur indicator [32], it is convenient to associate -1 to a loop rather than +1 (otherwise we can take extra care when bending lines). The ground state is therefore a superposition of loops, but weighted by $(-1)^{\text {number of loops }}$. This is commonly called the double-semion model.

There are two possible (gapped) boundaries for the toric code, the 'smooth' boundary, corresponding to the algebra $A_{0}$, and the 'rough' boundary, corresponding to $A_{1}$. We 
(a)

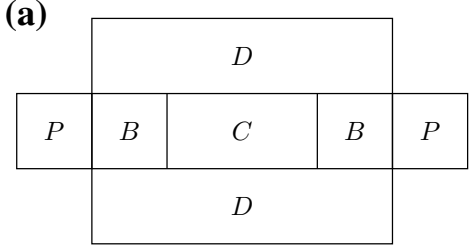

(b)

\begin{tabular}{|l|l|l|l|l|}
\hline$P$ & $B$ & $C$ & $B$ & $P$ \\
\hline
\end{tabular}

Fig. 2. Example of subsystems that can be used to find topological entropies in $(2+1)$-dimensions. The region $A$ is the complement of $B C D$. The regions a) are used to find the bulk entropy $\gamma$, and the regions b) are used for the boundary entropy $\Gamma$

refer to Ref. [54] for more details. The double-semion model only allows for one kind of (gapped) boundary, labeled by $A_{0}$.

In (3+1)-dimensions, each of these models labels a distinct Walker-Wang model. The models $\operatorname{Vec}^{1,1}\left(\mathbb{Z}_{2}\right)$, Vec ${ }^{1,-1}\left(\mathbb{Z}_{2}\right)$ are commonly called the bosonic- and fermionic- toric code models respectively [26,55]. Since these categories both have $\mathcal{Z}_{2}(\mathcal{C})=\{1, x\}$, they both have a single particle excitation, in addition to the trivial excitation, whose selfstatistics lead to the names of the models. The two models $\operatorname{Vec}^{ \pm i}(-1)$ are both referred to as semion models.

In (3+1)-dimensions, the bosonic toric code still has two kinds of boundaries, but the remaining models are only compatible with the trivial boundary labeled by $A_{0}$.

\section{Entropy Diagnostics}

In what follows we describe the universal correction to the area law that we expect for topological phases. We then define two diagnostics that can be used to probe the properties of the excitations at the boundary of (3+1)-dimensional topological phases.

4.1. The universal correction to the area law. The ground states of topological phases of matter demonstrate robust long-range entanglement that is not present in trivial phases [15-17]. Typically, we expect the entanglement entropy shared between a subsystem of a ground state of a gapped phase with the rest of the system to respect an area law, i.e., the entanglement will scale with the size of the surface area of the subsystem. The longrange entanglement manifests as a universal correction to the area law. More precisely, we expect that if we partition the ground state of a system into two subsystems, $R$ and its complement $R^{c}$, the entanglement entropy, $S_{R}$, will satisfy

$$
S_{R}=\alpha|\partial R|-b_{R} \gamma
$$

Here $\alpha$ is a non-universal coefficient that depends on the microscopic details of the system, $|\partial R|$ is the surface area of the interface between the partitioned regions, $b_{R}$ is the number of disjoint components of the interface between $R$ and $R^{c}$, and $\gamma$ is a universal constant commonly known as the topological entanglement entropy. We have assumed that $R$ is large compared to the correlation length of the system, and its shape has no irregular features. 
4.2. $(2+1)$-dimensional models. Intimately connected to the long-range entanglement of a topological phase are the properties of its low-energy excitations. A large class of topological models in (2+1)-dimensions are the Levin-Wen string-net models [9]. These models support topological point-like excitations that can be braided to change the state of the system.

Throughout this work we will be interested in the boundaries of topological phases. Importantly, topological particles can behave differently in the vicinity of the boundary of a phase. For instance, topological particles found in the bulk may become trivial particles close to certain boundaries. This is because topological particles can condense at the boundary such that non-trivial charges can be created locally.

As the physics of the quasi-particles of a topological phase can change close to its boundary, so to do we expect that the nature of its long-range entanglement to change. In Ref. [29], several topological entanglement entropy diagnostics were found to probe long-range entanglement of a model, both in the bulk and near to a boundary. The first is the bulk topological entanglement entropy

$$
\gamma:=S_{B C}+S_{C D}-S_{B}-S_{D}
$$

where the regions are depicted in Fig. 2a, and $X Y:=X \cup Y$. If $\gamma=0$, all point-like excitations can be created on the distinct parts of $P$ with a creation operator that has no support on $A C D$, where $A$ is the region that is complement to those shown in the figure. In this case, we declare them trivial. Conversely, if there are non-trivial topological excitations, for example created with string-like operators, $\gamma$ is necessarily non-zero.

In the presence of a gapped boundary, the excitations may differ. If a bulk topological excitation can be discarded or 'condensed' on the boundary, it is possible to locally create such an excitation near the boundary. This is detected using the diagnostic

$$
\Gamma:=S_{B C}+S_{C D}-S_{B}-S_{D}
$$

where the regions are depicted in Fig. 2b. If $\Gamma=0$, all point-like excitations on $P$ can be created with an operator that has no support on $A C D$, while non-trivial excitations require non-zero $\Gamma$.

4.3. $(3+1)$-dimensional models. Walker-Wang models give rise to both point- and linelike topological particles in the bulk, in addition to boundary excitations. Unlike LevinWen models, in some instances topological particles are only found at the boundary.

Since there are two kinds of topological excitations in $(3+1)$-dimensions, we might expect that there are two bulk diagnostics generalizing $\gamma$. However, as it has been shown $[25,28]$, these coincide. We define the bulk topological entanglement entropy

$$
\delta:=S_{B C}+S_{C D}-S_{B}-S_{D}
$$

where the regions are depicted in Fig. 3. We obtained this choice of region following intuition given in Ref. [29] where we consider the creating point excitations at the distinct parts of region $P$ using a string operator supported on $A C D$. We find $\delta$ is zero only if all the excitations can be created using an operator with local support. The boundary diagnostics that we describe next are obtained by bisecting the regions shown in Fig. 3 along different planes where the boundary lies.

In Ref. [29] two topological entanglement entropy diagnostics were found to probe long-range entanglement of a model near to a boundary. The first boundary diagnostic 


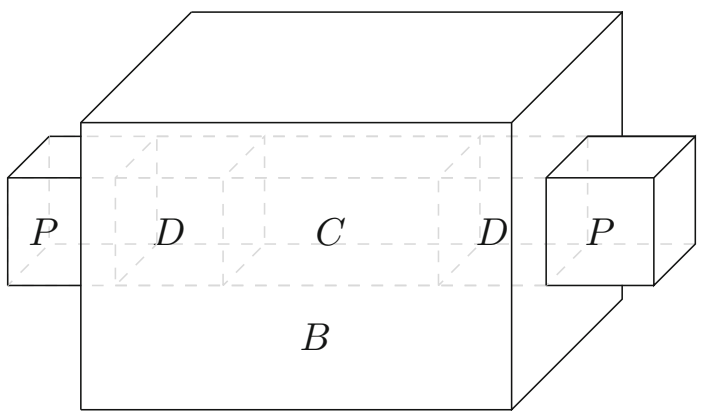

Fig. 3. Partitioning of the lattice for detecting excitations in the bulk. $B$ encircles $C D$, and $A$ is the complement of $B C D$. If $\delta$ is small, excitations on $P$ can be created by only acting on $P D$ and so have trivial statistics
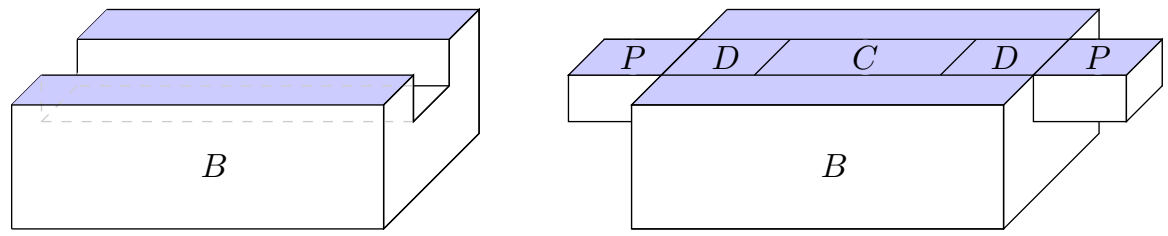

Fig. 4. Partitioning of the lattice for detecting point-like excitations on the boundary. The top (blue) surface is on the physical boundary of the lattice. If $\Delta_{\bullet}$ is small, excitations on $P$ can be created by only acting on $P D$ and so have trivial statistics

is an indicator that point-like topological particles can be created at the boundary of the system, and the second indicates that the boundary supports extended one-dimensional 'loop-like' topological particles. Unlike in the bulk, these diagnostics do not necessarily coincide. The first

$$
\Delta_{\bullet}:=S_{B C}+S_{C D}-S_{B}-S_{D},
$$

defined using the regions in Fig. 4, is non-zero if non-trivial point-like excitations can be created near the boundary. If $\Delta_{\bullet}=0$, all point-like excitations on $P$ can be created with a local operator, so they are necessarily trivial. Conversely, if there are non-trivial point-like particles near the boundary, $\Delta_{\bullet}>0$.

The final diagnostic is designed to detect nontrivial loop-like excitations. Using the regions depicted in Fig. 5, this diagnostic is

$$
\Delta_{\circ}:=S_{B C}+S_{C D}-S_{B}-S_{D} .
$$

Similarly to the other diagnostics, if $\Delta_{\circ}$ is zero, then line-like excitations must be trivial. Conversely, $\Delta_{\circ}$ must be nonzero if non-trivial loop excitations can be created at the boundary.

The diagnostics presented in Ref. [29] were found using generic arguments about the support of deformable operators that are used to create excitations. As such, it was shown rigorously that the null outcome is obtained only if a boundary does not give rise to topological particles. Conversely, a boundary that gives rise to topological excitations must give a positive reading for these diagnostics. However, due to spurious contributions [56-60], the generic arguments cannot guarantee that the diagnostics do not give false positives and, moreover, the work gives no interpretation for the magnitude of a positive reading. In our work, we restrict to loop-gas models. In that setting, for a large class of models, we obtain expressions for the topological entanglement entropy near the boundary. 

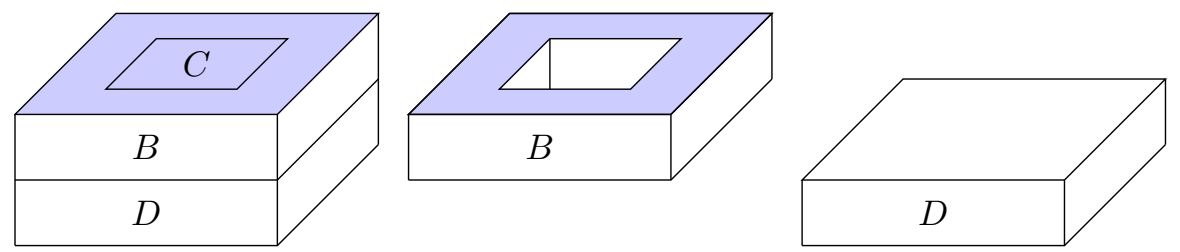

Fig. 5. Partitioning of the lattice for detecting line-like excitations on the boundary. The top (blue) surface is on the boundary of the lattice. If $\Delta_{\circ}$ is small, excitations on $B$ can be created without acting on $C$ and so have trivial statistics

\section{Bulk Entropy of Topological Loop-Gasses}

We now show how the entanglement entropy of ground states of Levin-Wen models is computed far from any boundary, before moving on to Walker-Wang models. To make the calculation we take the Schmidt decomposition of the ground state

$$
|\psi\rangle=\sum_{\lambda=1}^{r} \Phi_{\lambda}\left|\psi_{R}^{\lambda}\right\rangle\left|\psi_{R^{c}}^{\lambda}\right\rangle,
$$

for regions $R$, where the sets $\left\{\left|\psi_{R}^{\lambda}\right\rangle\right\}$ and $\left\{\left|\psi_{R^{c}}^{\lambda}\right\rangle\right\}$ are orthonormal, and $r$ is the Schmidt rank of the state $|\psi\rangle$. This allows us to compute the reduced state $\rho_{R}$ on $R$. Diagonalizing this matrix yields the entanglement entropy.

In what follows, we will need to parameterize the states $\left|\psi_{R}^{\lambda}\right\rangle$ and $\left|\psi_{R^{c}}^{\lambda}\right\rangle$. Recall from Sect. 3 that ground states of the loop-gas models can be understood as classes of diagrams which are related by local moves. It is convenient to parameterize $\left|\psi_{R}^{\lambda}\right\rangle$ in a similar way. Far from the interface (since the correlation length is 0 , far means one site), the state behaves exactly like the ground state. Unlike in the bulk, the interface defines a fixed boundary condition for the diagram in $R$. States $\left|\psi_{R}^{\lambda}\right\rangle$ will therefore be represented by some fiducial diagram $T$, and are understood to consist of a superposition of all diagrams that can be obtained from $T$ by local moves restricted to $R$ as indicated in Fig. 6. Particular lattices may provide geometric complications, but the topological invariance of the ground state will mean these are of no consequence. In all cases, we will choose a particular class of fusion trees as fiducial diagrams. For the following, we will therefore need several results concerning fusion trees. Consider fusing $n$ strings labeled $\vec{x}:=\left(x_{1}, x_{2}, \ldots, x_{n}\right)$ to a fixed object $a$. Using $F$-moves, we can bring the fusion tree for this process into the canonical form

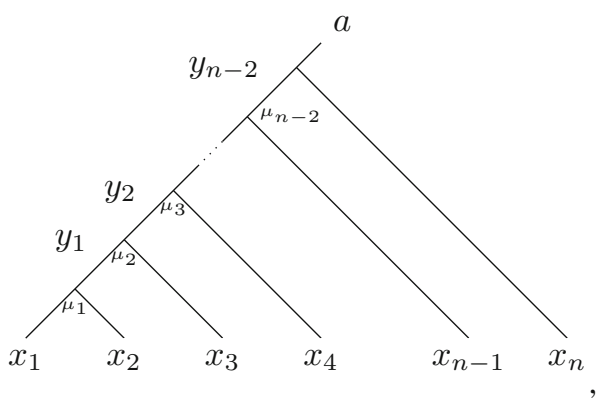


(a)

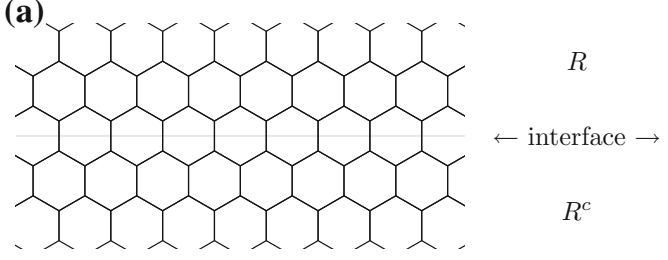

(b)

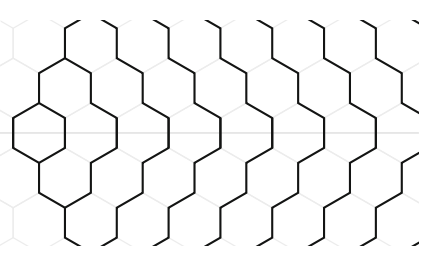

Fig. 6. To compute the Schmidt decomposition, we need to parameterize states contained on one sub-region. Given a generic configuration on the lattice (a), we can utilize the 'moves' outlined in Sect. 2, restricted to either side of the interface, to deform into a tree (b). The lattice sites on the boundary provide boundary conditions for the states in $R$ and $R^{c}$. We utilize constraints on the total fusion outcome in each region to parameterize the allowed trees

where $1 \leq \mu \leq N_{a, b}^{c}$ parameterizes the distinct fusion channels $a \otimes b \rightarrow c$. In the following, sums over $x_{i}, y_{i}$ are over all simple objects in $\mathcal{C}$. First, we need two results concerning summing over trees.

Lemma 2. Let $\mathcal{C}$ a unitary fusion category, then for a fixed simple fusion outcome a,

$$
\sum_{\vec{x}, \vec{y}} N_{x_{1} x_{2}}^{y_{1}} N_{y_{1} x_{3}}^{y_{2}} \ldots N_{y_{n-2} x_{n}}^{a} \prod_{j \leq n} d_{x_{j}}=d_{a} \mathcal{D}^{2(n-1)},
$$

where $\mathcal{D}=\sqrt{\sum_{i} d_{i}^{2}}$ is the total quantum dimension of $\mathcal{C}$.

Proof. Provided in Appendix B.

Lemma 3. Let $\mathcal{C}$ a unitary fusion category, then for a fixed simple fusion outcome a,

$$
\sum_{\vec{x}, \vec{y}} N_{x_{1} x_{2}}^{y_{1}} N_{y_{1} x_{3}}^{y_{2}} \ldots N_{y_{n-2} x_{n}}^{a} \frac{\prod_{j \leq n} d_{x_{j}}}{\mathcal{D}^{2(n-1)}} \log \prod_{k \leq n} d_{x_{k}}=n d_{a} \sum_{x} \frac{d_{x}^{2} \log d_{x}}{\mathcal{D}^{2}} .
$$

Proof. Provided in Appendix B.

Finally, we need the probability of a given fusion tree in a topological loop-gas model.

Lemma 4. (Probability of trees) Let $\mathcal{C}$ a unitary fusion category. Given a fusion outcome a on $n$ edges, the probability of the tree in Eq. (16) is

$$
\operatorname{Pr}[\vec{x}, \vec{y}, \vec{\mu} \mid a]=\frac{\prod_{j \leq n} d_{x_{j}}}{d_{a} \mathcal{D}^{2(n-1)}} .
$$

Proof. Provided in Appendix B.

Throughout the remainder of this section, we use the following condensed notation

$$
\begin{aligned}
\sum_{\vec{x}, \vec{y}, \vec{\mu}} & :=\sum_{x_{1}, \ldots, x_{n}} \sum_{y_{1}, \ldots, y_{n-2}} \sum_{\mu_{1}, \ldots, \mu_{n-2}} \sum_{x_{1}, \ldots, x_{n}} \sum_{y_{1}, \ldots, y_{n-2}}^{y_{x_{1} x_{2}} N_{y_{1} x_{3}}^{y_{2}} \ldots N_{y_{n-2} x_{n}}^{a},}
\end{aligned}
$$

where we frequently leave the fusion outcome $a$ implicit. 


\subsection{Levin-Wen models.}

Theorem 1 (Topological entropy of (2+1)-dimensional Levin-Wen models in the bulk $[16,17,61])$. Consider the regions shown in Fig. 2a, then the Levin-Wen model defined by a unitary spherical fusion category $\mathcal{C}$, with total dimension $\mathcal{D}$, has topological entropy

$$
\gamma=2 \log \mathcal{D}^{2}=\log \mathcal{D}_{\mathcal{Z}(\mathcal{C})}^{2}
$$

where $\mathcal{Z}(\mathcal{C})$ is the modular category called the Drinfeld center [31] of $\mathcal{C}$ which describes the anyons of the theory.

Examples. Recall the examples from Sect. 2.1. As discussed in Sect. 3.3, these label two distinct loop-gas models in $(2+1)$-dimensions, the toric code and double semion models. Since all the input categories for these examples have $\mathcal{D}^{2}=2$, the topological entanglement entropy is $\gamma=2 \log 2$ for both.

Lemma 5 (Entropy of (union of) simply connected bulk regions [17,28,61]). On a region $R$ in the bulk consisting of the disjoint union of simply connected sub-regions, the entropy is

$$
S_{R}=n S[\mathcal{C}]-b_{0} \log \mathcal{D}^{2},
$$

where $b_{0}$ is the number of disjoint interface components of $R, n$ is the number of links crossing the entanglement interface, and

$$
S[\mathcal{C}]:=\log \mathcal{D}^{2}-\sum_{x} \frac{d_{x}^{2} \log d_{x}}{\mathcal{D}^{2}} .
$$

Proof of Lemma 5. Consider a ball $R$ with $n$ sites along the interface, in the configuration $\vec{x}=x_{1}, x_{2}, \ldots, x_{n}$. Since any configuration must be created by inserting closed loops into the empty state, the total 'charge' crossing the interface must be 1 . For a fixed $\vec{x}$, there are now many ways for this to happen, parameterized by trees depicted in Eq. (16) with fusion outcome $a=1$.

Trees with distinct labelings (in $\vec{x}, \vec{y}$ or $\vec{\mu}$ ) are orthogonal. This means that if the tree Eq. (16) occurs adjacent to the interface within $R$, it must also occur on the other side of the interface

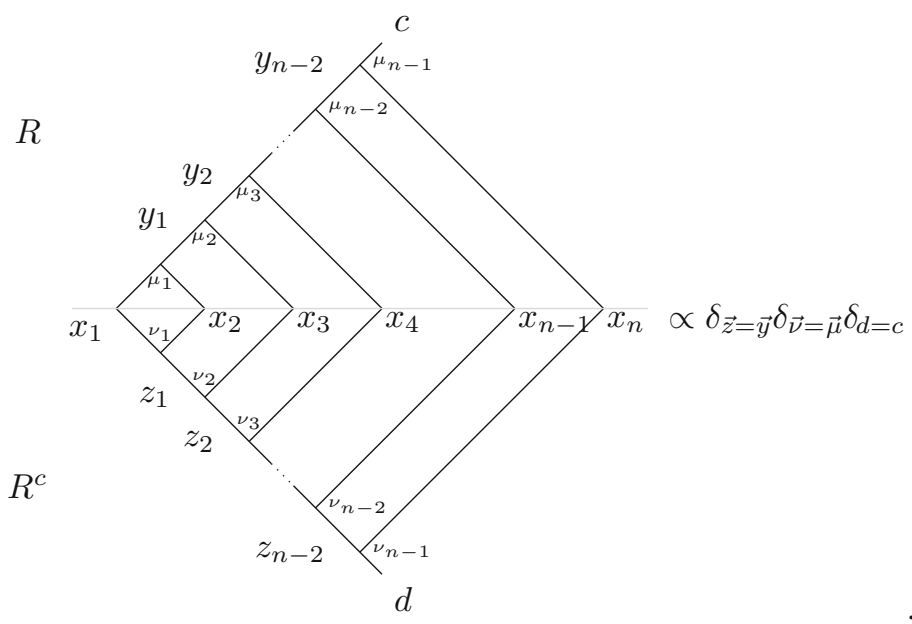


If the trees on either side of the cut had different branching structures, we could use local moves on either side of the cut to bring them to this standard form.

We take the Schmidt decomposition of the ground state as follows

$$
|\psi\rangle=\sum_{\vec{x}, \vec{y}, \vec{\mu}} \Phi_{\vec{x}, \vec{y}, \vec{\mu}}\left|\psi_{R}^{\vec{x}, \vec{y}, \vec{\mu}}\right\rangle\left|\psi_{R^{c}}^{\vec{x}, \vec{y}, \vec{\mu}}\right\rangle,
$$

where the notation $\vec{x}, \vec{y}, \vec{\mu}$ indicates the labeling of a valid tree as in Eq. (16). The state $\left|\psi_{R}^{\vec{x}, \vec{y}, \vec{\mu}}\right\rangle$ includes any state that can be reached from Eq. (16) (with $a=1$ ) by acting only on $R$. The reduced state on $R$ is

$$
\begin{aligned}
& \rho_{R}=\sum_{\vec{x}, \vec{y}, \vec{\mu}}\left|\Phi_{\vec{x}, \vec{y}, \vec{\mu}}\right|^{2}\left|\psi_{R}^{\vec{x}, \vec{y}, \vec{\mu}}\right\rangle\left\langle\psi_{R}^{\vec{x}, \vec{y}, \vec{\mu}}\right| \\
& =\sum_{\vec{x}, \vec{y}, \vec{\mu}} \operatorname{Pr}[\vec{x}, \vec{y}, \vec{\mu} \mid 1]\left|\psi_{R}^{\vec{x}, \vec{y}, \vec{\mu}}\right\rangle\left\langle\psi_{R}^{\vec{x}, \vec{y}, \vec{\mu}}\right|,
\end{aligned}
$$

where $\operatorname{Pr}[\vec{x}, \vec{y}, \vec{\mu} \mid 1]$ is the probability of the labeled tree, given that $\vec{x}$ fuses to 1 . From Lemma 4, the reduced state is

$$
\rho_{R}=\sum_{\vec{x}, \vec{y}, \vec{\mu}} \frac{\prod_{j \leq n} d_{x_{j}}}{\mathcal{D}^{2(n-1)}}\left|\psi_{R}^{\vec{x}, \vec{y}, \vec{\mu}}\right\rangle\left\langle\psi_{R}^{\vec{x}, \vec{y}, \vec{\mu}}\right| .
$$

The von Neumann entropy of $\rho_{R}$ is therefore

$$
\begin{aligned}
S_{R} & :=-\operatorname{tr} \rho_{R} \log \rho_{R} \\
& =-\sum_{\vec{x}, \vec{y}, \vec{\mu}} \frac{\prod_{j \leq n} d_{x_{j}}}{\mathcal{D}^{2(n-1)}} \log \frac{\prod_{k \leq n} d_{x_{k}}}{\mathcal{D}^{2(n-1)}} \\
& =\frac{\log \mathcal{D}^{2(n-1)}}{\mathcal{D}^{2(n-1)}} \sum_{\vec{x}, \vec{y}, \vec{\mu}} \prod_{j \leq n} d_{x_{j}}-\sum_{\vec{x}, \vec{y}, \vec{\mu}} \frac{\prod_{j \leq n} d_{x_{j}}}{\mathcal{D}^{2(n-1)}} \log \prod_{k \leq n} d_{x_{k}} \\
& =n\left(\log \mathcal{D}^{2}-\sum_{x} \frac{d_{x}^{2} \log d_{x}}{\mathcal{D}^{2}}\right)-\log \mathcal{D}^{2} \\
& =n S[C]-\log \mathcal{D}^{2}
\end{aligned}
$$

where Lemmas 2 and 3 are applied to the left and right terms of line (32), respectively, and

$$
S[C]:=\log \mathcal{D}^{2}-\sum_{x} \frac{d_{x}^{2} \log d_{x}}{\mathcal{D}^{2}} .
$$

It is straightforward to check that this holds on each sub-region of $R$.

Applying Lemma 5 to the regions in Fig. 2a completes the proof of Theorem 1. 
5.2. Walker-Wang models. In this section, we prove the following result for the bulk diagnostic for Walker-Wang models. The essential arguments in this section were made in Ref. [28], however we use slightly different language that allows the result to be applied more generally.

Theorem 2. For a Walker-Wang model defined by a unitary premodular category $\mathcal{C}$, the topological entanglement entropy (defined using the regions in Fig. 3) in the bulk is given by

$$
\delta=\sum_{c, \lambda_{c}} \frac{\lambda_{c}}{\mathcal{D}^{2}} \log \frac{\lambda_{c}}{d_{c}}
$$

where $\left\{\lambda_{c}\right\}$ are the eigenvalues of $\mathcal{S}_{c}^{\dagger} \mathcal{S}_{c}$, and $\mathcal{S}_{c}$ is the connected $\mathcal{S}$-matrix (Eq. (2)).

Proof. In simply connected regions, the arguments from Lemma 5 still hold. The other type of region in Fig. $2 \mathrm{a}$ is a torus. In this case, we cannot simply decompose the ground state as in Eq. (26), with the sum over configurations on the interface. Recall that the reason we could do this for a simple region was ground states are created by inserting closed loops, and all closed loops except those crossing the interface can be added entirely within either $R$ or $R^{c}$. This is not the case for a toroidal region. Consider, for example, the configuration depicted in Fig. 7. The closed string inside $R$ (red, dashed) cannot be altered by acting entirely within $R$, so contributes additional entanglement to the ground state, which is not witnessed by the interface configuration. Additionally, the two loops may be connected by a string, such that the global charge is trivial. Therefore, unlike for simply connected regions, the net charge crossing the boundary is not necessarily trivial. With these considerations, we can decompose the ground state as

$$
|\psi\rangle=\sum_{\substack{\vec{x}, \vec{y}, \vec{\mu} \\ c, a, \alpha, b, \beta}} \Phi_{\vec{x}, \vec{y}, \vec{\mu}, c} \frac{\left[\mathcal{S}_{c}\right]_{(b, \beta)(a, \alpha)}}{\mathcal{D}}\left|\psi_{R}^{\vec{x}, \vec{y}, \vec{\mu}, c, a, \alpha}\right\rangle\left|\psi_{R^{c}}^{\vec{x}, \vec{y}, \vec{\mu}, c, b, \beta}\right\rangle,
$$

where $\mathcal{S}_{c}$ is the connected $\mathcal{S}$-matrix defined in Eq. (2). The indices $\vec{x}, \vec{y}, \vec{\mu}$ are as in Eq. (26), $b$ labels the loop encircling $R$, while $a$ is the loop within $R$, and $c$ is the total charge crossing the boundary (the top label in Eq. (16)). The reduced state on $R$ is

$$
\begin{aligned}
\rho_{R} & =\sum_{\substack{\vec{x}, \vec{y}, \vec{\mu} \\
a_{1}, \alpha_{1}, a_{2}, \alpha_{2}, c}} \frac{\operatorname{Pr}[\vec{x}, \vec{y}, \vec{\mu} \mid c]}{\mathcal{D}^{2}}\left[\mathcal{S}_{c}^{\dagger} \mathcal{S}_{c}\right]_{\left(a_{2}, \alpha_{2}\right)\left(a_{1}, \alpha_{1}\right)}\left|\psi_{R}^{\vec{x}, \vec{y}, \vec{\mu}, a_{1}, \alpha_{1}, c}\right\rangle\left\langle\psi_{R}^{\vec{x}, \vec{y}, \vec{\mu}, a_{2}, \alpha_{2}, c}\right| \\
& =\sum_{\substack{\vec{x}, \vec{y}, \vec{\mu} \\
a_{1}, \alpha_{1}, a_{2}, \alpha_{2}, c}} \frac{\prod_{j \leq n} d_{x_{j}}}{d_{c} \mathcal{D}^{2 n}}\left[\mathcal{S}_{c}^{\dagger} \mathcal{S}_{c}\right]_{\left(a_{2}, \alpha_{2}\right)\left(a_{1}, \alpha_{1}\right)}\left|\psi_{R}^{\vec{x}, \vec{y}, \vec{\mu}, a_{1}, \alpha_{1}, c}\right\rangle\left\langle\psi_{R}^{\vec{x}, \vec{y}, \vec{\mu}, a_{2}, \alpha_{2}, c}\right| \cdot(39)
\end{aligned}
$$

To compute the entropy of this state, it is convenient to diagonalize it. Denote the eigenvalues of $\mathcal{S}_{c}^{\dagger} \mathcal{S}_{c}$ by $\left\{\lambda_{c}\right\}$. By a unitary change of basis, we have

$$
U \rho_{R} U^{\dagger}=\sum_{\substack{\vec{x}, \vec{y}, \vec{\mu}, c, \lambda_{c}}} \frac{\prod_{j \leq n} d_{x_{j}}}{d_{c} \mathcal{D}^{2 n}} \lambda_{c}\left|\varphi_{\lambda_{c}}^{\vec{x}, \vec{y}, \vec{\mu}, c}\right\rangle\left\langle\varphi_{\lambda_{c}}^{\vec{x}, \vec{y}, \vec{\mu}, c}\right|,
$$




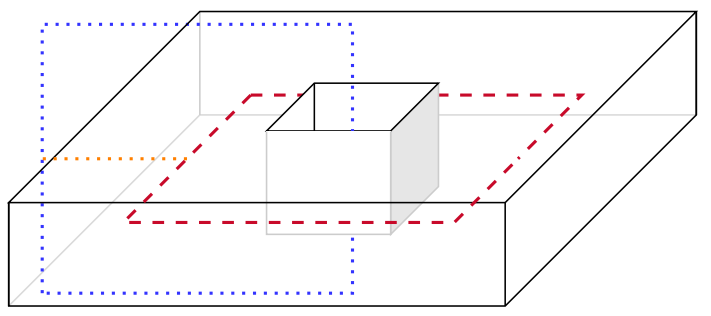

Fig. 7. When the region $R$ is not simply connected, the computation of entropy is more subtle. There is additional entanglement in the system due to intersecting loops that cannot be created in $R$ or $R^{c}$ separately. This is not witnessed by the configuration of strings on the interface

with von Neumann entropy

$$
S_{R}=n S[\mathcal{C}]-\sum_{c, \lambda_{c}} \frac{\lambda_{c}}{\mathcal{D}^{2}} \log \frac{\lambda_{c}}{d_{c}},
$$

where Lemmas 1 to 3 are used. Combining with Lemma 5 completes the proof.

Conjecture 1. Let $\mathcal{C}$ be a unitary premodular category $\mathcal{C}$, and define the connected $\mathcal{S}$-matrix via its matrix elements

$$
\left[\mathcal{S}_{c}\right]_{(a, \alpha),(b, \beta)}=\frac{1}{\mathcal{D}} \bar{a}(\bar{b}) \bar{c}
$$

The connected $\mathcal{S}$-matrix obeys

$$
\sum_{c, \lambda_{c}} \frac{\lambda_{c}}{\mathcal{D}^{2}} \log \frac{\lambda_{c}}{d_{c}}=\log \mathcal{D}_{\mathcal{Z}_{2}(\mathcal{C})}^{2}
$$

where $\left\{\lambda_{c}\right\}$ are the eigenvalues of $\mathcal{S}_{c}^{\dagger} \mathcal{S}_{c}$, and $\mathcal{Z}_{2}(\mathcal{C})$ is the Müger center of $\mathcal{C}$.

We conjecture that Eq. (43) holds in general, however we are currently unable to compute the spectrum of $\mathcal{S}_{c}$ beyond the families outlined in Theorem 3 .

Theorem 3. For a Walker-Wang model defined by a unitary premodular category of one of the following types:

- $\mathcal{C}=\mathcal{A} \otimes \mathcal{B}$, where $\mathcal{A}$ is symmetric and $\mathcal{B}$ is modular [28],

- $\mathcal{C}$ pointed,

- $\operatorname{rk}(\mathcal{C})<6$ and multiplicity free,

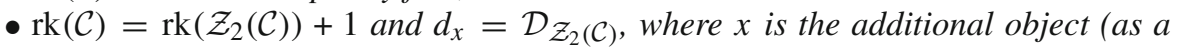
special case, $\mathcal{C}$ is a Tambara-Yamagami category [62, 63]), 
then Eq. (43) holds. As a consequence, the topological entanglement entropy (defined using the regions in Fig. 3) in the bulk is given by

$$
\delta=\log \mathcal{D}_{\mathcal{Z}_{2}(\mathcal{C})}^{2}
$$

where $\mathcal{Z}_{2}(\mathcal{C})$ is the Müger center of $\mathcal{C}$. As special cases, this includes

$$
\begin{aligned}
\delta_{\text {modular }} & =0 \\
\delta_{\text {symmetric }} & =\log \mathcal{D}^{2}
\end{aligned}
$$

We conjecture that Eq. (44) holds in generality. Physically, this is seen by noting that the particle content of the bulk Walker-Wang model is given by the Müger center $\mathcal{Z}_{2}(\cdot)$ [48].

Proof. Provided in Appendix B.

Examples. Recall the examples from Sect. 2.1. As discussed in Sect. 3.3, these label four distinct loop-gas models in (3+1)-dimensions, the bosonic and fermionic toric code models, and two semion models. All four input categories are pointed, so we can apply Theorem 3 to obtain the topological entanglement entropy. The first two models are symmetric, so $\delta=\log 2$ for both. The inputs to the semion models are modular, so the bulk is trivial [27]. In this case $\delta=0$.

\section{Boundary Entropy of Topological Loop-Gasses}

We now turn to the computation of the boundary diagnostics from Sect. 4. As before, we begin with Levin-Wen models.

\subsection{Levin-Wen models.}

Theorem 4. (Topological entropy of (2+1)-dimensional Levin-Wen models at a boundary) Consider the regions shown in Fig. 2b. The Levin-Wen model defined by unitary spherical fusion category $\mathcal{C}$, with boundary specified by an indecomposable $Q$-system $A \in \mathcal{C}$ has boundary entropy

$$
\Gamma=\log \mathcal{D}^{2}
$$

where $\mathcal{D}$ is the total quantum dimension of $\mathcal{C}$.

Examples. Recall the examples from Sect. 2.1. As discussed in Sect. 3.3, these label two distinct loop-gas models in (2+1)-dimensions, the toric code, and the double semion. The toric code has two possible boundary conditions, while the double semion only allows for the trivial boundary. All boundaries have $\Gamma=\log 2$.

Recall that a boundary for a Levin-Wen model defined by $\mathcal{C}$ is specified by an algebra object $A \in \mathcal{C}$. The algebra encodes the strings that can terminate on the boundary. This interpretation leads us to the following lemma. 
Lemma 6 (Entropy of (union of) simply connected regions, with boundary). On a region $R$ consisting of the disjoint union of simply connected sub-regions, the entropy is

$$
S_{R}=n S[\mathcal{C}]+\frac{b_{1}}{2} \log d_{A}-b_{0} \log \mathcal{D}^{2}
$$

where $b_{0}$ is the number of disjoint interface components of $R, b_{1}$ is the number of points where the entanglement surface intersects the physical boundary, and $n$ is the number of links crossing the entanglement interface.

Proof. Consider a ball $R$ with $n$ sites along the interface, which is in contact with the boundary. Recall that in the bulk, the fusion of the strings crossing the boundary was required to be 1 . In the presence of the boundary, this conservation rule is modified, since loops can terminate. All that is now required is that the fusion is in $A$

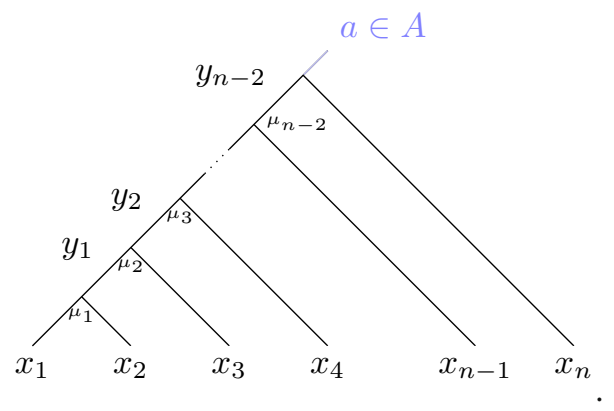

The ground state can be decomposed as

$$
|\psi\rangle=\sum_{\substack{\vec{x}, \vec{y}, \vec{\mu} \\ a \in A}} \Phi_{\vec{x}, \vec{y}, \vec{\mu}, a}\left|\psi_{R}^{\vec{x}, \vec{y}, \vec{\mu}, a}\right\rangle\left|\psi_{R^{c}}^{\vec{x}, \vec{y}, \vec{\mu}, a}\right\rangle .
$$

As before, the state $\left|\psi_{R}^{\vec{x}, \vec{y}, \vec{\mu}, a}\right\rangle$ includes any state that can be reached from Eq. (49) by acting only on $R$. The reduced state on $R$ is

$$
\begin{aligned}
\rho_{R} & =\sum_{\substack{\vec{x}, \vec{y}, \vec{\mu} \\
a \in A}}\left|\Phi_{\vec{x}, \vec{y}, \vec{\mu}, a}\right|^{2}\left|\psi_{R}^{\vec{x}, \vec{y}, \vec{\mu}, a}\right\rangle\left\langle\psi_{R}^{\vec{x}, \vec{y}, \vec{\mu}, a}\right| \\
& =\sum_{\substack{\vec{x}, \vec{y}, \vec{\mu} \\
a \in A}} \operatorname{Pr}[\vec{x}, \vec{y}, \vec{\mu} \mid a] \operatorname{Pr}[a]\left|\psi_{R}^{\vec{x}, \vec{y}, \vec{\mu}, a}\right\rangle\left\langle\psi_{R}^{\vec{x}, \vec{y}, \vec{\mu}, a}\right|,
\end{aligned}
$$

where $\operatorname{Pr}[\vec{x}, \vec{y}, \vec{\mu} \mid a]$ is the probability of the labeled tree, given that $\vec{x}$ fuses to $a$, and $\operatorname{Pr}[a \in A]=d_{a} / d_{A}$. Therefore,

$$
\rho_{R}=\sum_{\substack{\vec{x}, \vec{y}, \vec{\mu} \\ a \in A}} \frac{\prod_{j \leq n} d_{x_{j}}}{\mathcal{D}^{2(n-1)} d_{A}}\left|\psi_{R}^{\vec{x}, \vec{y}, \vec{\mu}, a}\right\rangle\left\langle\psi_{R}^{\vec{x}, \vec{y}, \vec{\mu}, a}\right| .
$$

Applying Lemmas 2 and 3 completes the proof for this region. It is straightforward to check that this holds on each sub-region of $R$, where Lemma 5 is used for any bulk sub-region. 


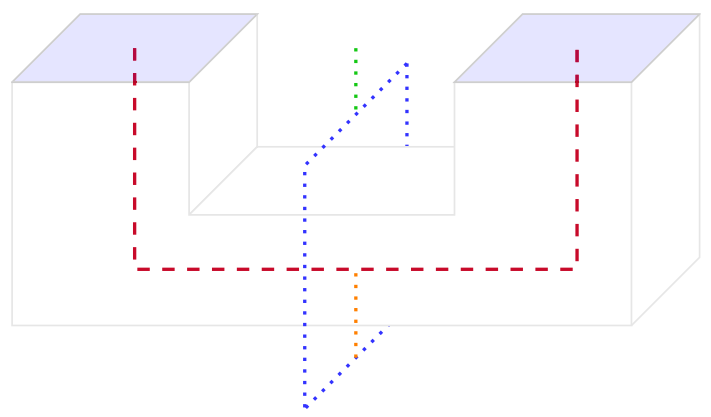

Fig. 8. When the region $R$ is in non-simple contact with a boundary on which strings can terminate, the computation of entropy is more subtle. There is additional entanglement in the system due to intersecting loops that cannot be created in $R$ (red, dashed) or $R^{c}$ (blue, dotted) separately. The red (internal) strings can terminate on the boundary. Also, the blue loop can emit a string which can terminate on the boundary

Applying Lemma 6 to the regions in Fig. 2b completes the proof of Theorem 4.

We can make sense of this halving of the entropy by considering folding the plane. Suppose we fold the model in Fig. 2a so that it resembles Fig. 2b. This turns the bulk of a model defined by $\mathcal{C}$ to a boundary of a model labeled by $\mathcal{C} \otimes \mathcal{C}^{\mathrm{op}}$. The quantum dimension of the folded theory is $\mathcal{D}_{\mathcal{C} \otimes \mathcal{C}^{\text {op }}}=\mathcal{D}_{\mathcal{C}}^{2}$, so the bulk diagnostic for $\mathcal{C}$ matches the boundary diagnostic computed for this folded theory.

6.2. Walker-Wang models. In (3+1)-dimensions, just like in $(2+1)$-dimensions, strings can terminate at the boundary. In addition, loops can interlock as discussed in Sect. 5.2. In the vicinity of the boundary, these two effects can occur simultaneously as depicted in Fig. 8.

For simply connected regions in contact with a boundary, we can apply Lemma 6 , replacing $b_{1} / 2$ with the number of lines where the region contacts the physical boundary. By applying the results so far, it is straightforward to check that the two diagnostics Eq. (13) and Eq. (14) are related by

$$
\Delta_{\circ}=\Delta_{\bullet}+\log d_{A}^{2}-\log \mathcal{D}^{2},
$$

so we only need to consider $\Delta_{\bullet}$. We are currently unable to compute this in general, however in this section we prove the following results:

Theorem 5. For a Walker-Wang model defined by a unitary premodular category $\mathcal{C}$, the entropy diagnostic $\Delta_{\bullet}$ for a boundary labeled by an indecomposable, commutative $Q$-system $A$ is given by

$$
\Delta_{\bullet}= \begin{cases}\log \mathcal{D}^{2} & A=1, \\ \log \mathcal{D}^{2}-\log d_{A} & \mathcal{C} \text { symmetric, } \\ \log \mathcal{D}^{2}-2 \log d_{A} & \mathcal{C} \text { pointed and } \mathcal{Z}_{2}(\mathcal{C}) \cap A=\{1\} . \text { In particular } \mathcal{C} \text { modular } . \\ \log \mathcal{D}^{2}-\log d_{A} & \mathcal{C} \text { pointed and } \mathcal{Z}_{2}(\mathcal{C}) \cap A=A .\end{cases}
$$

Examples. Recall the examples from Sect. 2.1. As discussed in Sect. 3.3, these label four distinct loop-gas models in $(3+1)$-dimensions, the bosonic and fermionic toric code 
models, and two semion models. All four input categories are pointed, so we can apply Theorem 5 to obtain the boundary topological entanglement entropy.

The bosonic toric code is compatible with two distinct gapped boundary conditions, labeled by $A_{0}$ and $A_{1}$ (see Sect. 2.1), with $d_{A_{0}}=d_{1}=1$, and $d_{A_{1}}=d_{1}+d_{x}=2$. Since the input category is symmetric, $\mathcal{Z}_{2}(\mathcal{C}) \cap A_{i}=A_{i}$, so the entropy diagnostics are $\Delta_{\bullet}\left(A_{0}\right)=\log 2-\log 1=\log 2$ and $\Delta_{\bullet}\left(A_{1}\right)=\log 2-\log 2=0$.

For the remaining examples, only the boundary labeled by $A_{0}$ is compatible, and $\Delta_{\bullet}=\log 2$ in all cases.

Proof. To capture configurations like the one in Fig. 8, we need new boundary $\mathcal{S}$-matrices resembling

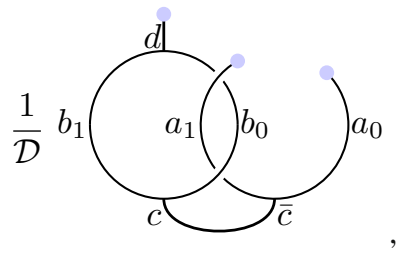

where the dots indicate where a string meets the boundary. We use boundary retriangulation invariance, as defined in Refs. [11,12] to evaluate this diagram on the ground space. Using this, we define the new $\mathcal{S}$-matrix elements as

$$
\left[\mathcal{S}_{c, d}\right]_{\left(b_{0}, b_{1}\right),\left(a_{0}, a_{1}\right)}:=\frac{m_{a_{1}, a_{0}}^{\bar{d}}}{\left(d_{a_{0}} d_{a_{1}} d_{d}\right)^{1 / 4} d_{A} \mathcal{D}} b_{1}
$$

where $a_{0}, a_{1}, d \in A$ and $b_{0}, b_{1}, c \in \mathcal{C}$. With this, the ground state can be written

$$
|\psi\rangle=\sum_{\substack{\vec{x}, \vec{y}, \vec{\mu} \\ c, b_{0}, b_{1} \in \mathcal{C} \\ d, a_{0}, a_{1} \in A}} \Phi_{\vec{x}, \vec{y}, \vec{\mu}, c} \frac{\left[\mathcal{S}_{c, d}\right]_{\left(b_{0}, b_{1}\right),\left(a_{0}, a_{1}\right)}}{N_{A}}\left|\psi_{R}^{\vec{x}, \vec{y}, \vec{\mu}, c, a_{0}, a_{1}}\right\rangle\left|\psi_{R^{c}}^{\vec{x}, \vec{y}, \vec{\mu}, c, b_{0}, b_{1}, d}\right\rangle,
$$

where $N_{A}$ is a normalizing factor. The reduced state on $R$ is

$$
\rho_{R}=\sum_{\substack{\vec{x}, \vec{y}, \vec{\mu} \\ c \in \mathcal{C} \\ d, a_{0}, a_{1}, a_{2}, a_{3} \in A}} \frac{\prod_{j \leq n} d_{x_{j}}\left[\mathcal{S}_{c, d}^{\dagger} \mathcal{S}_{c, d}\right]_{\left(a_{2}, a_{3}\right),\left(a_{0}, a_{1}\right)}}{d_{c} \mathcal{D}^{2(n-1)} N_{A}}\left|\psi_{R}^{\vec{x}, \vec{y}, \vec{\mu}, c, a_{0}, a_{1}}\right\rangle\left\langle\psi_{R}^{\vec{x}, \vec{y}, \vec{\mu}, c, a_{2}, a_{3}}\right|
$$


6.2.1. $A=1$ When the algebra is trivial, no strings can terminate. In that case, $\mathcal{S}_{1,1}^{\dagger} \mathcal{S}_{1,1}=1$, so the reduced state is

$$
\rho_{R}=\sum_{\vec{x}, \vec{y}, \vec{\mu}} \frac{\prod_{j \leq n} d_{x_{j}}}{\mathcal{D}^{2(n-1)}}\left|\psi_{R}^{\vec{x}, \vec{y}, \vec{\mu}}\right\rangle\left\langle\psi_{R}^{\vec{x}, \vec{y}, \vec{\mu}}\right|,
$$

which is diagonal and has entropy

$$
S_{R}=n S[C]-\log \mathcal{D}^{2} .
$$

6.2.2. $\mathcal{C}$ symmetric When $\mathcal{C}$ is symmetric, the rings in Eq. (57) separate, so

$$
\begin{aligned}
{\left[\mathcal{S}_{c, d}\right]_{\left(b_{0}, b_{1}\right),\left(a_{0}, a_{1}\right)} } & =\delta_{c=d} \sqrt{d_{b_{0}} d_{b_{1}}} \frac{\left(d_{a_{0}} d_{a_{1}}\right)^{1 / 4} m_{a_{1}, a_{0}}^{\bar{d}}}{d_{d}^{1 / 4} d_{A}^{2} \mathcal{D}}, \\
{\left[\mathcal{S}_{c, d}^{\dagger} \mathcal{S}_{c, d}\right]_{\left(a_{2}, a_{3}\right),\left(a_{0}, a_{1}\right)} } & =\delta_{c=d} \sum_{b_{0}, b_{1}} N_{b_{0} b_{1}}^{d} \frac{d_{b_{0}} d_{b_{1}}}{\sqrt{d_{d}}}\left(d_{a_{0}} d_{a_{1}} d_{a_{2}} d_{a_{3}}\right)^{1 / 4} \frac{m_{a_{1}, a_{0}}^{\bar{d}}\left(m_{a_{3}, a_{2}}^{\bar{d}}\right)^{*}}{d_{A}^{4} \mathcal{D}^{2}} \\
& =\delta_{c=d}\left(d_{a_{0}} d_{a_{1}} d_{a_{2}} d_{a_{3}}\right)^{1 / 4} \sqrt{d_{d}} \frac{m_{a_{1}, a_{0}}^{\bar{d}}\left(m_{a_{3}, a_{2}}\right)^{*}}{d_{A}^{2}} .
\end{aligned}
$$

It can readily be verified that this matrix is rank 1 . The eigenvalue can be found using Eq. (5), giving $\lambda=d_{d} / d_{A}$. We can therefore write the state on $R$ as

$$
\begin{aligned}
\rho_{R} & =\sum_{\substack{\vec{x}, \vec{y}, \vec{\mu} \\
c \in \mathcal{C} \\
d \in A}} \delta_{c=d} \frac{\prod_{j \leq n} d_{x_{j}}}{\mathcal{D}^{2(n-1)} d_{A}}\left|\psi_{R}^{\vec{x}, \vec{y}, \vec{\mu}, c}\right\rangle\left\langle\psi_{R}^{\vec{x}, \vec{y}, \vec{\mu}, c}\right| \\
& =\sum_{\substack{\vec{x}, \vec{y}, \vec{\mu} \\
c \in A}} \frac{\prod_{j \leq n} d_{x_{j}}}{\mathcal{D}^{2(n-1)} d_{A}}\left|\psi_{R}^{\vec{x}, \vec{y}, \vec{\mu}, c}\right\rangle\left\langle\psi_{R}^{\vec{x}, \vec{y}, \vec{\mu}, c}\right| .
\end{aligned}
$$

Using Lemmas 2 and 3, we find that the entropy of this state is

$$
\mathcal{S}_{R}=n S[\mathcal{C}]-\log \mathcal{D}^{2}+\log d_{A}
$$

6.2.3. $\mathcal{C}$ pointed When all quantum dimensions are equal to 1 , the boundary $\mathcal{S}$-matrix is

$$
\left[\mathcal{S}_{c, d}\right]_{\left(b_{0}, b_{1}\right),\left(a_{0}, a_{1}\right)}=\delta_{c=d} \frac{m_{a_{1}, a_{0}}^{\bar{d}}}{d_{A}}\left[\mathcal{S}_{1}\right]_{b_{0}, \bar{a}_{1}},
$$

where $\left[\mathcal{S}_{1}\right]_{b_{0}, a_{1}}$ is the $\mathcal{S}$-matrix from Eq. (2). This gives

$$
\left[\mathcal{S}_{c, d}^{\dagger} \mathcal{S}_{c, d}\right]_{\left(a_{2}, a_{3}\right),\left(a_{0}, a_{1}\right)}=\delta_{c=d} \frac{m_{a_{1}, a_{0}}^{\bar{d}}\left(m_{a_{3}, a_{2}}^{\bar{d}}\right)^{*}}{d_{A}^{2}} \sum_{b_{0}}\left[\mathcal{S}_{1}\right]_{b_{0}, \bar{a}_{3}}^{*}\left[\mathcal{S}_{1}\right]_{b_{0}, \bar{a}_{1}}
$$


Using Lemmas 2.4 and 2.13 of Ref. [38], this can be simplified to

$$
\left[\mathcal{S}_{c, d}^{\dagger} \mathcal{S}_{c, d}\right]_{\left(a_{2}, a_{3}\right),\left(a_{0}, a_{1}\right)}=\delta_{c=d} \frac{m_{a_{1}, a_{0}}^{\bar{d}}\left(m_{a_{3}, a_{2}}^{\bar{d}}\right)^{*}}{d_{A}^{2}} \delta_{a_{3} \otimes \bar{a}_{1} \in \mathcal{Z}_{2}(\mathcal{C})} .
$$

Pointed braided categories have fusion rules given by an Abelian group $G$ [31,64], and algebras are twisted group algebras $[65,66]$ of subgroups of $G$. Moreover, $\mathcal{Z}_{2}(\mathcal{C})$ also has fusion rules given by a subgroup.

Since $a_{3} \otimes \bar{a}_{1} \in A$ and $a_{3} \otimes \bar{a}_{1} \in \mathcal{Z}_{2}(\mathcal{C})$, there must be some $h \in \mathcal{Z}_{2}(\mathcal{C}) \cap A$ so that $a_{3}=a_{1} h$. We can then write

$$
\rho_{R}=\sum_{\substack{\vec{x} \\ d, a \in A \\ h \in \mathcal{Z}_{2}(\mathcal{C}) \cap A}} \frac{m_{a,(a d)^{-1}}^{d^{-1}}\left(m_{a h,(a h d)^{-1}}^{d^{-1}}\right)^{*}}{d_{A}^{2} \mathcal{D}^{2(n-1)} N_{A}}\left|\psi_{R}^{\vec{x}, d, a}\right\rangle\left\langle\psi_{R}^{\vec{x}, d, a h}\right|
$$

In the case that $\mathcal{Z}_{2}(\mathcal{C}) \cap A=A$, this reduces to the symmetric case, since summing over $h \in A$ is the same as summing over $A \ni h^{\prime}=a h$.

When $\mathcal{Z}_{2}(\mathcal{C}) \cap A=\{1\}$, the unit, the reduced state on $R$ simplifies to

$$
\rho_{R}=\sum_{\substack{\vec{x} \\ d, a \in A}} \frac{m_{a,(a d)^{-1}}^{d^{-1}}\left(m_{a,(a d)^{-1}}^{d^{-1}}\right)^{*}}{d_{A}^{2} \mathcal{D}^{2(n-1)} N_{A}}\left|\psi_{R}^{\vec{x}, d, a}\right\rangle\left\langle\psi_{R}^{\vec{x}, d, a}\right|
$$

This reduced state is diagonal, and has entropy

$$
\begin{aligned}
S_{R} & =-\sum_{\substack{\vec{x} \\
d, a \in A}} \frac{\mid m_{a,(a d)^{-1}}^{d^{-1}}}{\mathcal{D}^{2(n-1)} d_{A}^{2}} \log \left(\frac{\mid m_{a,(a d)^{-1}}^{d^{-1}}}{\mathcal{D}^{2(n-1)} d_{A}^{2}}\right) \\
& =-\sum_{\substack{\vec{x} \\
d, a \in A}} \frac{\mid m_{a,(a d)^{-1}}^{d^{-1}}}{\mathcal{D}^{2(n-1)} d_{A}^{2}} \log \left(\mid m_{a,(a d)^{-1}}^{d^{-1}}\right)+\log \left(\mathcal{D}^{2(n-1)} d_{A}^{2}\right),
\end{aligned}
$$

where we have made use of Eq. (5). Since $A$ is a twisted group algebra, we may assume $\left|m_{a b}^{c}\right| \in\{0,1\}$. Finally, this gives

$$
\begin{aligned}
S_{R} & =\log \left(\mathcal{D}^{2(n-1)} d_{A}^{2}\right) \\
& =S[\mathcal{C}]-\log \mathcal{D}^{2}+2 \log d_{A},
\end{aligned}
$$

completing the proof. 


\section{Remarks}

To summarize, we have evaluated the long-range entanglement in the bulk, and at the boundary, of (2+1)- and (3+1)-dimensional topological phases. In (2+1)-dimensions, we found the entropy diagnostic $\Gamma=\log \mathcal{D}^{2}$ regardless of the choice of boundary algebra $A$. This is in contrast to the results for (3+1)-dimensions, where a signature of the boundary, namely its dimension as an algebra, can be seen in the diagnostics $\Delta_{\bullet}$ and $\Delta_{\circ}$.

The most natural boundary for these models is defined by the algebra $A=1$, which (uniquely) always exists. At this boundary, we found that the point-like diagnostic $\Delta$. recovers the total dimension of the input category. In particular, when $\mathcal{C}$ is a $(2+1)$ dimensional anyon model, this is consistent with a boundary that supports the anyons. Conversely, the loop-like diagnostic $\Delta_{\circ}$ is zero at these boundaries, ruling out loop-like excitations in the vicinity.

We have conjectured a general property of the connected $\mathcal{S}$-matrix which, if proven in general, allows computation of bulk Walker-Wang topological entropy. Such a proof may also be interesting for the classification of premodular categories in general. To the best of our knowledge, there is no complete classification of boundaries for WalkerWang models. Such a classification is complicated by requiring, as a sub-classification, a complete understanding of (2+1)-dimensional theories. This goes beyond the scope of the current work, and we have therefore specialized to boundaries described by Qsystems and to particular families of input fusion category. Extending these results may provide a more complete understanding of the possible boundary excitations and their properties.

Acknowledgements. We particularly thank David Aasen for useful discussions, and informing us of the class of Walker-Wang boundaries we consider. We are also grateful to Taiga Adair, Daniel Barter, Isaac Kim, Sam Roberts, Thomas Smith and Dominic Williamson for helpful discussions. We also thank Stephen Bartlett and Céline Bœhm. Research at Perimeter Institute is supported in part by the Government of Canada through the Department of Innovation, Science and Industry Canada and by the Province of Ontario through the Ministry of Colleges and Universities. This work is supported by the Australian Research Council (ARC) via the Centre of Excellence in Engineered Quantum Systems (EQuS) project number CE170100009. BJB was previously supported by the University of Sydney Fellowship Programme. SJE also received support from the EPSRC.

Open Access This article is licensed under a Creative Commons Attribution 4.0 International License, which permits use, sharing, adaptation, distribution and reproduction in any medium or format, as long as you give appropriate credit to the original author(s) and the source, provide a link to the Creative Commons licence, and indicate if changes were made. The images or other third party material in this article are included in the article's Creative Commons licence, unless indicated otherwise in a credit line to the material. If material is not included in the article's Creative Commons licence and your intended use is not permitted by statutory regulation or exceeds the permitted use, you will need to obtain permission directly from the copyright holder. To view a copy of this licence, visit http://creativecommons.org/licenses/by/4.0/.

Publisher's Note Springer Nature remains neutral with regard to jurisdictional claims in published maps and institutional affiliations.

\section{Appendix A: Properties of the Connected $\mathcal{S}$-matrix}

Proposition A.1 (Premodular trap). Let $\mathcal{C}$ be a unitary premodular category, then

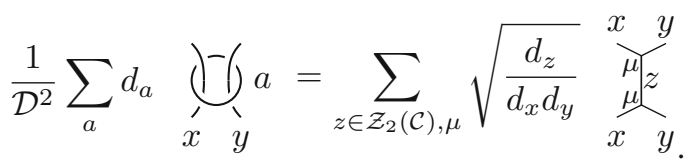


Proof. This is a slight generalization of Proposition 3.1 of Ref. [67], following from Lemmas 2.4 and 2.13 of Ref. [38].

Lemma 1. Let $\mathcal{C}$ be a unitary premodular category, then

$$
\sum_{c \in \mathcal{C}} \operatorname{Tr} \mathcal{S}_{c}^{\dagger} \mathcal{S}_{c}=\mathcal{D}^{2},
$$

where $\mathcal{S}_{c}$ is the connected $\mathcal{S}$-matrix and $\mathcal{D}$ is the total dimension of $\mathcal{C}$.

Proof. The operator $\mathcal{S}_{c}$ acts on the fusion spaces as [32]

$$
\mathcal{S}_{c} \gamma_{\beta}^{\bar{b}}=\frac{\sqrt{d_{c}}}{\mathcal{D}} \sum_{x} d_{x} \overbrace{c}^{\bar{x}} \bar{b} .
$$

Using Eq. (A3), we have

$$
\sum_{c} \operatorname{Tr} \mathcal{S}_{c}^{\dagger} \mathcal{S}_{c}=\sum_{a, \alpha, c}\left[\mathcal{S}_{c}^{\dagger} \mathcal{S}_{c}\right]_{(a, \alpha),(a, \alpha)}
$$

using the properties of the trace. Applying the premodular trap (Proposition A.1), this gives

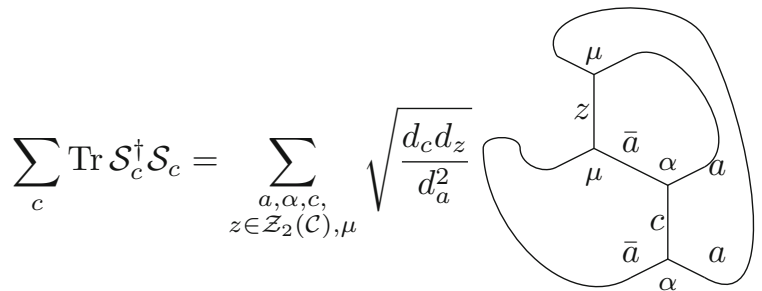




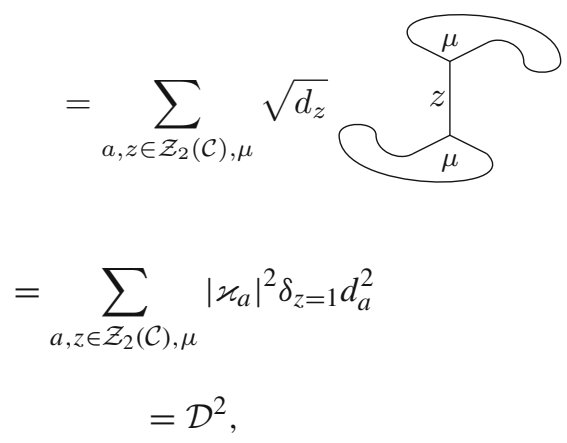

where $\varkappa_{a}$ is the Frobenius-Schur indicator [32].

\section{Appendix B: Loop-Gas Results}

In this section, given a fusion category $\mathcal{C}$, an $n$-tuple of simple objects $\vec{x}_{n}:=\left(x_{1}, x_{2}, \ldots\right.$, $x_{n}$ ), and a fixed simple object $a$, we use the notation

$$
N_{a}\left(\vec{x}_{n}\right):=\sum_{\vec{y}_{n-2}} N_{x_{1}, x_{2}}^{y_{1}} N_{y_{1}, x_{3}}^{y_{2}} \ldots N_{y_{n-2}, x_{n}}^{a},
$$

where $\vec{y}_{n-2}:=\left(y_{1}, y_{2}, \ldots, y_{n-2}\right)$, and the sum is over all tuples of simple objects in $\mathcal{C}$. $N_{a}\left(\vec{x}_{n}\right)$ counts the number of ways $\vec{x}_{n}$ can fuse to $a$. When it can easily be inferred, we omit the subscript on the tuple $\vec{x}$.

Lemma 2. Let $\mathcal{C}$ be a unitary fusion category, then

$$
\sum_{\vec{x}_{n}} N_{a}\left(\vec{x}_{n}\right) \prod_{j \leq n} d_{x_{j}}=d_{a} \mathcal{D}^{2(n-1)},
$$

where $\mathcal{D}=\sqrt{\sum_{a} d_{a}^{2}}$ is the total quantum dimension of $\mathcal{C}$.

Proof. We proceed inductively.

When $n=1, N_{a}(x)=\delta_{x=a}=N_{1, x}^{a}$, and Eq. (B2) reduces to $d_{a}=d_{a}$.

Assume Eq. (B2) holds for the fusion of $n$ objects. Recall that or any fusion category, we have

$$
d_{a} d_{b}=\sum_{c} N_{b c}^{a} d_{c}
$$

and this holds for any cyclic permutation of the indices on $N_{b c}^{a}$. We now obtain

$$
\begin{aligned}
\sum_{\vec{x}_{n+1}} N_{a}\left(\vec{x}_{n+1}\right) \prod_{j \leq n+1} d_{x_{j}} & =\sum_{\vec{x}_{n}, y_{n-1}} N_{y_{n-1}}\left(\vec{x}_{n}\right) \prod_{j \leq n} d_{x_{j}} \sum_{x_{n+1}} N_{y_{n-1}, x_{n+1}}^{a} d_{x_{n+1}} \\
& =\mathcal{D}^{2(n-1)} \sum_{x_{n+1}, y_{n-1}} N_{y_{n-1}, x_{n+1}}^{a} d_{y_{n-1}} d_{x_{n+1}} \\
& =\mathcal{D}^{2(n-1)} d_{a} \sum_{y_{n-1}} d_{y_{n-1}}^{2} \\
& =d_{a} \mathcal{D}^{2 n}
\end{aligned}
$$

where in the second line we used the induction assumption (Eq. (B2)), and in the third line we used Eq. (B3). 
Lemma 3. Let $\mathcal{C}$ a unitary fusion category. For the fusion of $n$ objects $\vec{x}=$ $\left(x_{1}, x_{2}, \ldots, x_{n}\right)$, with $n>1$, we have

$$
\sum_{\vec{x}_{n}} N_{a}\left(\vec{x}_{n}\right) \frac{\prod_{j \leq n} d_{x_{j}}}{\mathcal{D}^{2(n-1)}} \log \prod_{k \leq n} d_{x_{k}}=n d_{a} \sum_{x} \frac{d_{x}^{2} \log d_{x}}{\mathcal{D}^{2}} .
$$

Proof. We prove the claim inductively. The base case is when $n=2$.

$$
\begin{aligned}
& \sum_{x_{1}, x_{2}} N_{x_{1}, x_{2}}^{a} \frac{d_{x_{1}} d_{x_{2}}}{\mathcal{D}^{2}}\left(\log d_{x_{1}}+\log d_{x_{2}}\right) \\
& =\sum_{x_{1}, x_{2}} N_{x_{1}, x_{2}}^{a} \frac{d_{x_{1}} d_{x_{2}}}{\mathcal{D}^{2}} \log d_{x_{1}}+\sum_{x_{1}, x_{2}} N_{x_{1}, x_{2}}^{a} \frac{d_{x_{1}} d_{x_{2}}}{\mathcal{D}^{2}} \log d_{x_{2}} \\
& =d_{a} \sum_{x_{1}} \frac{d_{x_{1}}^{2}}{\mathcal{D}^{2}} \log d_{x_{1}}+d_{a} \sum_{x_{2}} \frac{d_{x_{2}}^{2}}{\mathcal{D}^{2}} \log d_{x_{2}} \\
& =2 d_{a} \sum_{x} \frac{d_{x}^{2} \log d_{x}}{\mathcal{D}^{2}} .
\end{aligned}
$$

Assume Eq. (B8) holds for $n$-tuples, then

$$
\begin{aligned}
\sum_{\vec{x}_{n}, x_{n+1}} N_{a}\left(\vec{x}_{n+1}\right) \frac{\prod_{j \leq n+1} d_{x_{j}}}{\mathcal{D}^{2 n}} \log \prod_{k \leq n+1} d_{x_{k}} \\
=\sum_{\substack{\vec{x}_{n} \\
y_{n-1}, x_{n+1}}} N_{y_{n-1}}\left(\vec{x}_{n}\right) N_{y_{n-1}, x_{n+1}} \frac{\prod_{j \leq n} d_{x_{j}}}{\mathcal{D}^{2 n}} d_{x_{n+1}} \log \left(\prod_{k \leq n} d_{x_{k}} d_{n+1}\right) \\
=\sum_{\vec{x}_{n}} N_{y_{n-1}}\left(\vec{x}_{n}\right) \frac{\prod_{j \leq n} d_{x_{j}}}{\mathcal{D}^{2 n}} N_{y_{n-1}, x_{n+1}}^{a} d_{x_{n+1}}\left(\log \prod_{k \leq n} d_{x_{k}}+\log d_{x_{n+1}}\right) \\
=n \sum_{x} \frac{d_{x}^{2} \log d_{x}}{\mathcal{D}^{2}} \sum_{y_{n-1}, x_{n+1}} \frac{N_{y_{n-1}, x_{n+1}}^{a} d_{y_{n-1}} d_{x_{n+1}}}{\mathcal{D}^{2}}+\sum_{y_{n-1}, x_{n+1}} N_{y_{n-1}, x_{n+1}}^{a} \frac{d_{y_{n-1}} d_{x_{n+1}} \log d_{x_{n+1}}}{\mathcal{D}^{2}} \\
=n d_{a} \sum_{x} \frac{d_{x}^{2} \log d_{x}}{\mathcal{D}^{2}} \sum_{x_{n+1}} \frac{d_{x_{n+1}}^{2}+d_{a} \sum_{x_{n+1}} \frac{d_{x_{n+1}}^{2} \log d_{x_{n+1}}}{\mathcal{D}^{2}}}{=} \\
=(n+1) d_{a} \sum_{x} \frac{d_{x}^{2} \log d_{x}}{\mathcal{D}^{2}} .
\end{aligned}
$$


Lemma 4. Let $\mathcal{C}$ a unitary fusion category. Given a fixed fusion outcome a on $n$ simple objects, the probability of the tree

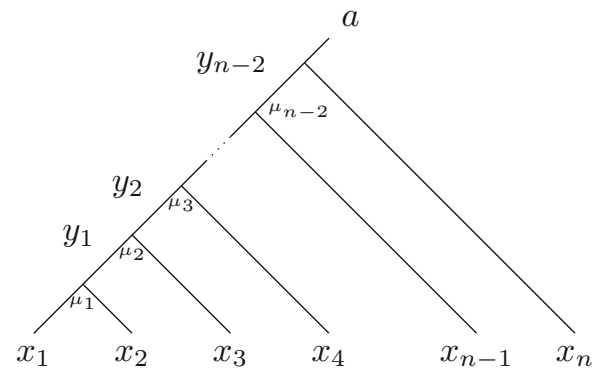

in the ground state of a topological loop-gas (Levin-Wen or Walker-Wang) model is

$$
\begin{aligned}
\operatorname{Pr}[\vec{x}, \vec{y}, \vec{\mu} \mid a] & =\frac{\prod_{j \leq n} \operatorname{Pr}\left[x_{j}\right]}{\operatorname{Pr}[a] \prod_{k \leq n} d_{x_{k}}} d_{a} \\
& =\frac{\prod_{j \leq n} d_{x_{j}}}{d_{a} \mathcal{D}^{2(n-1)}} .
\end{aligned}
$$

Proof. Given a pair of objects $a, b$, the probability that they fuse to $c$ is given by $[28,68]$

$$
\operatorname{Pr}[a \otimes b \rightarrow c]=\frac{N_{a b}^{c} d_{c}}{d_{a} d_{b}},
$$

so the probability that $x_{1} \otimes x_{2} \otimes \ldots \otimes x_{n} \rightarrow a$ is

$$
\begin{aligned}
\operatorname{Pr} & {\left[x_{1} \otimes x_{2} \otimes x_{3} \otimes \ldots \otimes x_{n} \rightarrow a\right] } \\
& =\sum_{\vec{y}} \operatorname{Pr}\left[x_{1} \otimes x_{2} \rightarrow y_{1}\right] \operatorname{Pr}\left[y_{1} \otimes x_{3} \rightarrow y_{2}\right] \cdots \operatorname{Pr}\left[y_{n-2} \otimes x_{n} \rightarrow a\right] \\
& =\frac{N_{a}(\vec{x})}{\prod_{j \leq n} d_{x_{j}}} d_{a},
\end{aligned}
$$

where

$$
\begin{aligned}
N_{a}(\vec{x}) & :=\sum_{\vec{y}} N_{x_{1} x_{2}}^{y_{1}} N_{y_{1} x_{3}}^{y_{2}} \ldots N_{y_{n-2} x_{n}}^{a} \\
& =\sum_{\vec{y}} N_{a}(\vec{x}, \vec{y}) .
\end{aligned}
$$

The probability of a configuration is

$$
\begin{aligned}
\operatorname{Pr}[\vec{x}, \vec{y} \mid a] & =\operatorname{Pr}\left[x_{1} \otimes x_{2} \otimes x_{3} \otimes \ldots \otimes x_{n} \rightarrow a\right] \frac{\prod_{j \leq n} \operatorname{Pr}\left[x_{j}\right]}{\operatorname{Pr}[a]} \\
& =\frac{N_{a}(\vec{x}, \vec{y}) d_{a}}{\prod_{k \leq n} d_{x_{k}}} \frac{\prod_{j \leq n} \operatorname{Pr}\left[x_{j}\right]}{\operatorname{Pr}[a]},
\end{aligned}
$$


where $\operatorname{Pr}\left[x_{i}\right]=d_{x_{i}}^{2} / \mathcal{D}^{2}$. For a fixed $\vec{x}$ and $\vec{y}$, all (allowed) $\vec{\mu}$ are equally likely, and there are $N_{a}(\vec{x}, \vec{y})$ such configurations, so

$$
\begin{aligned}
\operatorname{Pr}[\vec{x}, \vec{y}, \vec{\mu} \mid a] & =\frac{\prod_{j \leq n} \operatorname{Pr}\left[x_{j}\right]}{\operatorname{Pr}[a] \prod_{k \leq n} d_{x_{k}}} d_{a} \\
& =\frac{\prod_{j \leq n} d_{x_{j}}}{d_{a} \mathcal{D}^{2(n-1)}} .
\end{aligned}
$$

Lemma 2 can be used to show these are properly normalized.

Theorem 3. For a Walker-Wang model defined by a unitary premodular category of one of the following types:

1. $\mathcal{C}=\mathcal{A} \otimes \mathcal{B}$, where $\mathcal{A}$ is symmetric and $\mathcal{B}$ is modular [28],

2. $\mathcal{C}$ pointed,

3. $\operatorname{rk}(\mathcal{C})<6$ and multiplicity free,

4. $\operatorname{rk}(\mathcal{C})=\operatorname{rk}\left(\mathcal{Z}_{2}(\mathcal{C})\right)+1$ and $d_{x}=\mathcal{D}_{\mathcal{Z}_{2}(\mathcal{C})}$, where $x$ is the additional object,

then Eq. (43) holds. As a consequence, the topological entanglement entropy (defined using the regions in Fig. 3) in the bulk is given by

$$
\delta=\log \mathcal{D}_{\mathcal{Z}_{2}(\mathcal{C})}^{2}
$$

As special cases, this includes

$$
\begin{aligned}
\delta_{\text {modular }} & =0 \\
\delta_{\text {symmetric }} & =\log \mathcal{D}^{2}
\end{aligned}
$$

Proof.

B.1. Case 1. Using the premodular trap (Proposition A.1), we have the matrix elements of $\mathcal{S}_{c}^{\dagger} \mathcal{S}_{c}$

$$
\left[\mathcal{S}_{c}^{\dagger} \mathcal{S}_{c}\right]_{(a, \alpha),(b, \beta)}=\sqrt{\frac{d_{c}}{d_{a} d_{b}}} \sum_{x \in \mathcal{Z}_{2}(\mathcal{C}), \mu} \sqrt{d_{x}}\left(\begin{array}{ccc}
a & b
\end{array}\right.
$$

If $\mathcal{C}$ is symmetric, $\mathcal{Z}_{2}(\mathcal{C})=\mathcal{C}$, and

$$
\left[\mathcal{S}_{c}^{\dagger} \mathcal{S}_{c}\right]_{(a, \alpha),(b, \beta)}=\delta_{c=1} d_{a} d_{b}
$$

This matrix is rank 1 , with eigenvalue $\mathcal{D}^{2}$. If $\mathcal{C}$ is modular, $\mathcal{Z}_{2}(\mathcal{C})=$ Vec, and

$$
\left[\mathcal{S}_{c}^{\dagger} \mathcal{S}_{c}\right]_{(a, \alpha),(b, \beta)}=\delta_{a=b} \delta_{\alpha=\beta} \delta_{\bar{a} \otimes a=c} d_{c} .
$$

For fixed $c$, this matrix is rank $\sum_{c} N_{\bar{a}, a}^{c}$, with all eigenvalues equal to $d_{c}$. 
B.2. Case 2. If $\mathcal{C}$ is pointed (every simple object has dimension 1), then $\mathcal{S}_{c}=0$ unless $c=1$. In this case, the fusion rules are given by a finite Abelian group $A[31,64]$, and $\mathcal{Z}_{2}(\mathcal{C})=A^{\prime}$ has fusion rules given by a subgroup. From Lemmas 2.4 and 2.13 of Ref. [38], along with symmetries of the $\mathcal{S}_{1}$ matrix proven in Ref. [32] we know that

$$
\left[\mathcal{S}_{1}^{\dagger} \mathcal{S}_{1}\right]_{a b}=\sum_{c \in \mathcal{Z}_{2}(\mathcal{C})} N_{a \bar{b}}^{c} d_{c}
$$

so

$$
\left[\mathcal{S}_{1}^{\dagger} \mathcal{S}_{1}\right]_{a b}=1 \Longleftrightarrow a \in b A^{\prime}
$$

Therefore, $\left[\mathcal{S}_{1}^{\dagger} \mathcal{S}_{1}\right]$ is a block matrix, with $\left[A: A^{\prime}\right]=|A| /\left|A^{\prime}\right|$ blocks, labeled by the cosets of $A^{\prime}$, each full of ones. Therefore, there are $\left[A: A^{\prime}\right]$ eigenvalues, identically $\mathcal{D}_{\mathcal{Z}_{2}(\mathcal{C})}^{2}$. The entropy is given by

$$
\delta=\log \mathcal{D}_{\mathcal{Z}_{2}(\mathcal{C})}^{2}
$$

B.3. Case 3. Case 3 is proven explicitly in the attached Mathematica file [42]. Classification of the fusion rings for ranks 2-5 can be found in Ref. [67,69-72], along with Ref. [73]. Additionally, all multiplicity free fusion rings for ranks 1-6 can be found at Ref. [74]. From this, explicit $F$ and $R$ data can be found. The list of categories, along with their properties, is included beginning on Page 29.

B.4. Case 4. It is straightforward to check that if $a$ or $b$ are in $\mathcal{Z}_{2}(\mathcal{C})$, then

$$
\left[\mathcal{S}_{c}^{\dagger} \mathcal{S}_{c}\right]_{(a, \alpha),(b, \beta)}=\delta_{c=1} d_{a} d_{b} \delta_{a \in \mathcal{Z}_{2}(\mathcal{C})} \delta_{b \in \mathcal{Z}_{2}(\mathcal{C})},
$$

so $\mathcal{S}_{c}^{\dagger} \mathcal{S}_{c}$ has the form

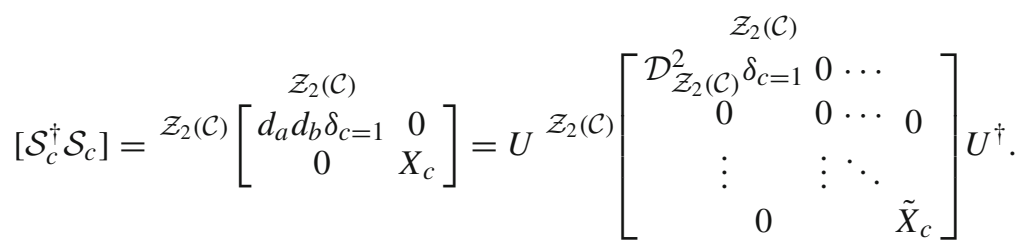

From the top left block, we have an eigenvector of $\mathcal{S}_{1}^{\dagger} \mathcal{S}_{1}$ with entries $v_{a}=\delta_{a \in \mathcal{Z}_{2}(\mathcal{C})} d_{a}$ with eigenvalue $\mathcal{D}_{\mathcal{Z}_{2}(\mathcal{C})}^{2}$. From Lemmas 2.4 and 2.13 of Ref. [38], along with symmetries of the $\mathcal{S}_{1}$ matrix proven in Ref. [32] we know that

$$
\left[\mathcal{S}_{1}^{\dagger} \mathcal{S}_{1}\right]_{a b}=\sum_{c \in \mathcal{Z}_{2}(\mathcal{C})} N_{a \bar{b}}^{c} d_{c} .
$$

The vector with entries $w_{a}=d_{a}$ is also an eigenvector with the same eigenvalue:

$$
\sum_{b \in \mathcal{C}} \sum_{c \in \mathcal{Z}_{2}(\mathcal{C})} N_{a \bar{b}}^{c} d_{c} d_{b}=\sum_{c \in \mathcal{Z}_{2}(\mathcal{C})} d_{a} d_{c}^{2}
$$




$$
=\mathcal{D}_{\mathcal{Z}_{2}(\mathcal{C})}^{2} d_{a}
$$

so we have an orthogonal vector $w-v$ with eigenvalue $\mathcal{D}_{\mathcal{Z}_{2}(\mathcal{C})}^{2}$.

If $\operatorname{rk}(\mathcal{C})=\operatorname{rk}\left(\mathcal{Z}_{2}(\mathcal{C})\right)+1$ and the additional object has $d_{x}=\mathcal{D}_{\mathcal{Z}_{2}(\mathcal{C})}$, then all other eigenvalues must be 0 since $\operatorname{Tr} \mathcal{S}_{1}^{\dagger} S_{1}=2 \mathcal{D}_{\mathcal{Z}_{2}(\mathcal{C})}^{2}$ and $\mathcal{D}^{2}=\mathcal{D}_{\mathcal{Z}_{2}(\mathcal{C})}^{2}+d_{x}^{2}$. The entropy of the Walker-Wang model in the bulk is

$$
\delta=\log \mathcal{D}_{\mathcal{Z}_{2}(\mathcal{C})}^{2} .
$$

B.5. Small category data. Data for small categories. "Valid" indicates that the pentagon, hexagon, and ribbon equations, along with unitarity, are true. "TY" indicates that the category has the property defined in Case 4 of Theorem 3.

Full data, including explicit $F$ and $R$ symbols is provided in the attached Mathematica files, also available at Ref. [42]. Note that these may take $a$ very long time to check. This is due to the complicated algebraic integers occurring, and Mathematica needing to simplify using the functions "Simplify" and "RootReduce".

Categories are named $\mathrm{FR}_{c ; x}^{a, b}$ according to their fusion ring $\mathrm{FR}_{c}^{a, b}$ from Ref. [74], along with their categorification ID $x$. Highlighted categories do not fall within any of

\begin{tabular}{|c|c|c|c|c|c|c|c|c|c|c|}
\hline Cat. ID & Rank & $\mathcal{D}^{2}$ & Valid & $\operatorname{rk}\left(\mathcal{Z}_{2}(\mathcal{C})\right)$ & Premodular & Pointed? & TY? & TEE & $\log \mathcal{D}_{\mathcal{Z}_{2}(\mathcal{C})}^{2}$ & Conjecture true? \\
\hline $\mathrm{FR}_{1,0}^{2,0}$ & 2 & 2 & $\begin{array}{ll} \\
\end{array}$ & 2 & Symm. & $\checkmark$ & & $\log 2$ & $\log 2$ & $\checkmark$ \\
\hline $\mathrm{FR}_{1: 1}^{2,0}$ & 2 & 2 & $\checkmark$ & 1 & Mod. & $\checkmark$ & $\checkmark$ & 0 & 0 & $\checkmark$ \\
\hline $\mathrm{FR}_{1: 2}^{2,0}$ & 2 & 2 & $\checkmark$ & 2 & Symm. & $\checkmark$ & & $\log 2$ & $\log 2$ & $\checkmark$ \\
\hline $\mathrm{FR}_{1.3}^{2,0}$ & 2 & 2 & 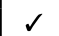 & 1 & Mod. & 2 & $\checkmark$ & 0 & 0 & 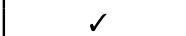 \\
\hline $\mathrm{FR}_{20.0}^{2,0}$ & 2 & $\frac{1}{2}(\sqrt{5}+5)$ & 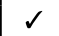 & 1 & Mod. & & & 0 & 0 & $\checkmark$ \\
\hline $\mathrm{FR}_{2 ; 1}^{2,0}$ & 2 & $\frac{1}{2}(\sqrt{5}+5)$ & $\checkmark$ & 1 & Mod. & & & 0 & 0 & $\checkmark$ \\
\hline $\mathrm{FR}_{1 ; 0}^{3,0}$ & 3 & 4 & $\sqrt{ }$ & 1 & Mod. & & & 0 & 0 & $\sqrt{ }$ \\
\hline $\mathrm{FR}_{1: 1}^{3,0}$ & 3 & 4 & 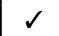 & 1 & Mod. & & & 0 & 0 & $\checkmark$ \\
\hline $\mathrm{FR}_{1.2}^{3,0}$ & 3 & 4 & $d$ & 1 & Mod. & & & 0 & 0 & $d$ \\
\hline $\mathrm{FR}_{1.3}^{3,0}$ & 3 & 4 & $\checkmark$ & 1 & Mod. & & & 0 & 0 & $s$ \\
\hline $\mathrm{FR}_{1: 4}^{3,0}$ & 3 & 4 & $\checkmark$ & 1 & Mod. & & & 0 & 0 & $\checkmark$ \\
\hline $\mathrm{FR}_{1,5}^{3,0}$ & 3 & 4 & $s$ & 1 & Mod. & & & 0 & 0 & $s$ \\
\hline $\mathrm{FR}_{1,6}^{3,0}$ & 3 & 4 & $s$ & 1 & Mod. & & & 0 & 0 & $s$ \\
\hline $\mathrm{FR}_{17}^{3,0}$ & 3 & 4 & 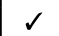 & 1 & Mod. & & & 0 & 0 & $d$ \\
\hline $\mathrm{FR}_{2,0}^{3,0}$ & 3 & 6 & $\checkmark$ & 3 & Symm. & & & $\log 6$ & $\log 6$ & $s$ \\
\hline $\mathrm{FR}_{2,1}^{3,0}$ & 3 & 6 & $\checkmark$ & 2 & $\checkmark$ & & & $\log 2$ & $\log 2$ & $\checkmark$ \\
\hline $\mathrm{FR}_{2,2}^{3,0}$ & 3 & 6 & $\checkmark$ & 2 & $s$ & & & $\log 2$ & $\log 2$ & $\checkmark$ \\
\hline $\mathrm{FR}_{3 ; 0}^{3,0}$ & 3 & $\sim 9.30$ & $\checkmark$ & 1 & Mod. & & & 0 & 0 & $\checkmark$ \\
\hline $\mathrm{FR}_{3,1}^{3,0}$ & 3 & $\sim 9.30$ & $\checkmark$ & 1 & Mod. & & & 0 & 0 & $\checkmark$ \\
\hline $\mathrm{FR}_{1 ; 0}^{3,2}$ & 3 & 3 & $\checkmark$ & 3 & Symm. & $\checkmark$ & & $\log 3$ & $\log 3$ & $\checkmark$ \\
\hline $\mathrm{FR}_{1 ; 1}^{3,2}$ & 3 & 3 & $\checkmark$ & 1 & Mod. & $\checkmark$ & & 0 & 0 & $\checkmark$ \\
\hline $\mathrm{FR}_{1 ; 2}^{3,2}$ & 3 & 3 & $\checkmark$ & 1 & Mod. & $\checkmark$ & & 0 & 0 & $\checkmark$ \\
\hline
\end{tabular}
the other cases in Theorem 3. 


\begin{tabular}{|c|c|c|c|c|c|c|c|c|c|c|}
\hline Cat. ID & Rank & $\bar{D}^{2}$ & Valid & $\overline{\operatorname{rk}\left(\mathcal{Z}_{2}(\mathcal{C})\right)}$ & Premodular? & Pointed? & TY? & TEE & $\log \mathcal{D}_{\mathcal{Z}_{2}(\mathcal{C})}^{2}$ & Conjecture true? \\
\hline $\mathrm{FR}_{1 ; 0}^{4,0}$ & 4 & 4 & $\checkmark$ & 4 & Symm. & $\checkmark$ & & $\log 4$ & $\log 4$ & $\checkmark$ \\
\hline $\mathrm{FR}_{1 ; 1}^{4,0}$ & 4 & 4 & $\checkmark$ & 1 & Mod. & $\checkmark$ & & 0 & 0 & $\checkmark$ \\
\hline $\mathrm{FR}_{1 ; 2}^{4,1}$ & 4 & 4 & $\checkmark$ & 4 & Symm. & $\checkmark$ & & $\log 4$ & $\log 4$ & $\checkmark$ \\
\hline $\mathrm{FR}_{1 ; 3}^{4,0}$ & 4 & 4 & $\checkmark$ & 1 & Mod. & $\checkmark$ & & 0 & 0 & $\checkmark$ \\
\hline $\mathrm{FR}_{1 ; 4}^{1,0}$ & 4 & 4 & $\checkmark$ & 2 & $\checkmark$ & $\checkmark$ & & $\log 2$ & $\log 2$ & $\checkmark$ \\
\hline $\mathrm{FR}_{1 ; 5}^{4,0}$ & 4 & 4 & $\checkmark$ & 2 & $\checkmark$ & $\checkmark$ & & $\log 2$ & $\log 2$ & $\checkmark$ \\
\hline $\mathrm{FR}_{1 ; 6}^{4,0}$ & 4 & 4 & $\checkmark$ & 2 & $\checkmark$ & $\checkmark$ & & $\log 2$ & $\log 2$ & $\checkmark$ \\
\hline $\mathrm{FR}_{1 ; 7}^{4,0}$ & 4 & 4 & $\checkmark$ & 2 & $\checkmark$ & $\checkmark$ & & $\log 2$ & $\log 2$ & $\checkmark$ \\
\hline $\mathrm{FR}_{1 ; 8}^{4,0}$ & 4 & 4 & $\checkmark$ & 4 & Symm. & $\checkmark$ & & $\log 4$ & $\log 4$ & $\checkmark$ \\
\hline $\mathrm{FR}_{1 ; 9}^{1,8}$ & 4 & 4 & $\checkmark$ & 1 & Mod. & $\checkmark$ & & 0 & 0 & $\checkmark$ \\
\hline $\mathrm{FR}_{1 ; 10}^{4,0}$ & 4 & 4 & $\checkmark$ & 4 & Symm. & $\checkmark$ & & $\log 4$ & $\log 4$ & $\checkmark$ \\
\hline $\mathrm{FR}_{1 ; 11}^{4,0}$ & 4 & 4 & $\checkmark$ & 1 & Mod. & $\checkmark$ & & 0 & 0 & $\checkmark$ \\
\hline $\mathrm{FR}_{1 ; 12}^{4,0}$ & 4 & 4 & $\checkmark$ & 2 & $\checkmark$ & $\checkmark$ & & $\log 2$ & $\log 2$ & $\checkmark$ \\
\hline $\mathrm{FR}_{1 ; 13}^{4,0}$ & 4 & 4 & $\checkmark$ & 2 & $\checkmark$ & $\checkmark$ & & $\log 2$ & $\log 2$ & $\checkmark$ \\
\hline $\mathrm{FR}_{1 ; 14}^{4,0}$ & 4 & 4 & $\checkmark$ & 2 & $\checkmark$ & $\checkmark$ & & $\log 2$ & $\log 2$ & $\checkmark$ \\
\hline $\mathrm{FR}_{1 ; 15}^{4,0}$ & 4 & 4 & $\checkmark$ & 2 & $\checkmark$ & $\checkmark$ & & $\log 2$ & $\log 2$ & $\checkmark$ \\
\hline $\mathrm{FR}_{1 ; 16}^{1,0}$ & 4 & 4 & $\checkmark$ & 1 & Mod. & $\checkmark$ & & 0 & 0 & $\checkmark$ \\
\hline $\mathrm{FR}_{1 ; 17}^{4,0}$ & 4 & 4 & $\checkmark$ & 2 & $\checkmark$ & $\checkmark$ & & $\log 2$ & $\log 2$ & $\checkmark$ \\
\hline $\mathrm{FR}_{1 ; 18}^{4,0}$ & 4 & 4 & $\checkmark$ & 1 & Mod. & $\checkmark$ & & 0 & 0 & $\checkmark$ \\
\hline $\mathrm{FR}_{1 ; 19}^{4,0}$ & 4 & 4 & $\checkmark$ & 2 & $\checkmark$ & $\checkmark$ & & $\log 2$ & $\log 2$ & $\checkmark$ \\
\hline $\mathrm{FR}_{1 ; 20}^{4,0}$ & 4 & 4 & $\checkmark$ & 1 & Mod. & $\checkmark$ & & 0 & 0 & $\checkmark$ \\
\hline $\mathrm{FR}_{1 ; 21}^{4,0}$ & 4 & 4 & $\checkmark$ & 1 & Mod. & $\checkmark$ & & 0 & 0 & $\checkmark$ \\
\hline $\mathrm{FR}_{1 ; 22}^{4,0}$ & 4 & 4 & $\checkmark$ & 1 & Mod. & $\checkmark$ & & 0 & 0 & $\checkmark$ \\
\hline $\mathrm{FR}_{1 ; 23}^{4,0}$ & 4 & 4 & $\checkmark$ & 1 & Mod. & $\checkmark$ & & 0 & 0 & $\checkmark$ \\
\hline $\mathrm{FR}_{1 ; 24}^{4,0}$ & 4 & 4 & $\checkmark$ & 1 & Mod. & $\checkmark$ & & 0 & 0 & $\checkmark$ \\
\hline $\mathrm{FR}_{1 ; 25}^{4,0}$ & 4 & 4 & $\checkmark$ & 2 & $\checkmark$ & $\checkmark$ & & $\log 2$ & $\log 2$ & $\checkmark$ \\
\hline $\mathrm{FR}_{1 ; 26}^{4,0}$ & 4 & 4 & $\checkmark$ & 1 & Mod. & $\checkmark$ & & 0 & 0 & $\checkmark$ \\
\hline $\mathrm{FR}_{1 ; 27}^{4,0}$ & 4 & 4 & $\checkmark$ & 2 & $\checkmark$ & $\checkmark$ & & $\log 2$ & $\log 2$ & $\checkmark$ \\
\hline $\mathrm{FR}_{1 ; 28}^{4,0}$ & 4 & 4 & $\checkmark$ & 1 & Mod. & $\checkmark$ & & 0 & 0 & $\checkmark$ \\
\hline $\mathrm{FR}_{1 ; 29}^{4,0}$ & 4 & 4 & $\checkmark$ & 1 & Mod. & $\checkmark$ & & 0 & 0 & $\checkmark$ \\
\hline $\mathrm{FR}_{1 ; 0}^{4,0}$ & 4 & 4 & $\checkmark$ & 1 & Mod. & $\checkmark$ & & 0 & 0 & $\checkmark$ \\
\hline $\mathrm{FR}_{1 ; 31}^{4,0}$ & 4 & 4 & $\checkmark$ & 1 & Mod. & $\checkmark$ & & 0 & 0 & $\checkmark$ \\
\hline $\mathrm{FR}_{2 ; 0}^{4,0}$ & 4 & $\sqrt{5}+5$ & $\checkmark$ & 2 & $\checkmark$ & & & $\log 2$ & $\log 2$ & $\checkmark$ \\
\hline $\mathrm{FR}_{2 ; 1}^{4,0}$ & 4 & $\sqrt{5}+5$ & $\checkmark$ & 1 & Mod. & & & 0 & 0 & $\checkmark$ \\
\hline $\mathrm{FR}_{2 ; 2}^{4,0}$ & 4 & $\sqrt{5}+5$ & $\checkmark$ & 2 & $\checkmark$ & & & $\log 2$ & $\log 2$ & $\checkmark$ \\
\hline $\mathrm{FR}_{2,3}^{4,0}$ & 4 & $\sqrt{5}+5$ & $\checkmark$ & 1 & Mod. & & & 0 & 0 & $\checkmark$ \\
\hline $\mathrm{FR}_{2 ; 4}^{4,0}$ & 4 & $\sqrt{5}+5$ & $\checkmark$ & 2 & $\checkmark$ & & & $\log 2$ & $\log 2$ & $\checkmark$ \\
\hline $\mathrm{FR}_{2 ; 5}^{4,0}$ & 4 & $\sqrt{5}+5$ & $\checkmark$ & 1 & Mod. & & & 0 & 0 & $\checkmark$ \\
\hline $\mathrm{FR}_{2 ; 6}^{4,0}$ & 4 & $\sqrt{5}+5$ & $\checkmark$ & 2 & $\checkmark$ & & & $\log 2$ & $\log 2$ & $\checkmark$ \\
\hline $\mathrm{FR}_{2 ; 7}^{4,0}$ & 4 & $\sqrt{5}+5$ & $\checkmark$ & 1 & Mod. & & & 0 & 0 & $\checkmark$ \\
\hline $\mathrm{FR}_{3 ; 0}^{4,0}$ & 4 & 10 & $\checkmark$ & 4 & Symm. & & & $\log 10$ & $\log 10$ & $\checkmark$ \\
\hline $\mathrm{FR}_{3 ; 1}^{4,0}$ & 4 & 10 & $\checkmark$ & 2 & $\checkmark$ & & & $\log 2$ & $\log 2$ & $\checkmark$ \\
\hline $\mathrm{FR}_{3 ; 2}^{4,0}$ & 4 & 10 & $\checkmark$ & 2 & $\checkmark$ & & & $\log 2$ & $\log 2$ & $\checkmark$ \\
\hline $\mathrm{FR}_{3 ; 3}^{4,0}$ & 4 & 10 & $\checkmark$ & 2 & $\checkmark$ & & & $\log 2$ & $\log 2$ & $\checkmark$ \\
\hline $\mathrm{FR}_{3 ; 4}^{4,0}$ & 4 & 10 & $\checkmark$ & 2 & $\checkmark$ & & & $\log 2$ & $\log 2$ & $\checkmark$ \\
\hline $\mathrm{FR}_{4 ; 0}^{4,0}$ & 4 & $4 \sqrt{2}+8$ & $\checkmark$ & 2 & $\checkmark$ & & & $\log 2$ & $\log 2$ & $\checkmark$ \\
\hline $\mathrm{FR}_{4 ; 1}^{4,0}$ & 4 & $4 \sqrt{2}+8$ & $\checkmark$ & 2 & $\checkmark$ & & & $\log 2$ & $\log 2$ & $\checkmark$ \\
\hline $\mathrm{FR}_{5 ; 0}^{4,0}$ & 4 & $\frac{1}{2}(5 \sqrt{5}+15)$ & $\checkmark$ & 1 & Mod. & & & 0 & 0 & $\checkmark$ \\
\hline $\mathrm{FR}_{5 ; 1}^{4,0}$ & 4 & $\frac{1}{2}(5 \sqrt{5}+15)$ & $\checkmark$ & 1 & Mod. & & & 0 & 0 & $\checkmark$ \\
\hline $\mathrm{FR}_{5 ; 2}^{4,0}$ & 4 & $\frac{1}{2}(5 \sqrt{5}+15)$ & $\checkmark$ & 1 & Mod. & & & 0 & 0 & $\checkmark$ \\
\hline $\mathrm{FR}_{5 ; 3}^{4,0}$ & 4 & $\frac{1}{2}(5 \sqrt{5}+15)$ & $\checkmark$ & 1 & Mod. & & & 0 & 0 & $\checkmark$ \\
\hline $\mathrm{FR}_{6 ; 0}^{4,0}$ & 4 & $\sim 19.24$ & $\checkmark$ & 1 & Mod. & & & 0 & 0 & $\checkmark$ \\
\hline $\mathrm{FR}_{6 ; 1}^{4,0}$ & 4 & $\sim 19.24$ & $\checkmark$ & 1 & Mod. & & & 0 & 0 & $\checkmark$ \\
\hline $\mathrm{FR}_{1 ; 0}^{4, \frac{1}{4,2}}$ & 4 & 4 & $\checkmark$ & 4 & Symm. & $\checkmark$ & & $\log 4$ & $\log 4$ & $\checkmark$ \\
\hline $\mathrm{FR}_{1 ; 1}^{1,0}$ & 4 & 4 & $\checkmark$ & 1 & Mod. & $\checkmark$ & & 0 & 0 & $\checkmark$ \\
\hline $\mathrm{FR}_{1 ; 2}^{4,2}$ & 4 & 4 & $\checkmark$ & 2 & $\checkmark$ & $\checkmark$ & & $\log 2$ & $\log 2$ & $\checkmark$ \\
\hline $\mathrm{FR}_{1 ; 3}^{4,2}$ & 4 & 4 & $\checkmark$ & 1 & Mod. & $\checkmark$ & & 0 & 0 & $\checkmark$ \\
\hline $\mathrm{FR}_{1 ; 4}^{1,2}$ & 4 & 4 & $\checkmark$ & 4 & Symm. & $\checkmark$ & & $\log 4$ & $\log 4$ & $\checkmark$ \\
\hline $\mathrm{FR}_{1 ; 5}^{1,2}$ & 4 & 4 & $\checkmark$ & 1 & Mod. & $\checkmark$ & & 0 & 0 & $\checkmark$ \\
\hline $\mathrm{FR}_{1 ; 6}^{4,2}$ & 4 & 4 & $\checkmark$ & 2 & $\checkmark$ & $\checkmark$ & & $\log 2$ & $\log 2$ & $\checkmark$ \\
\hline $\mathrm{FR}_{1 ; 7}^{4,2}$ & 4 & 4 & $\checkmark$ & 1 & Mod. & $\checkmark$ & & 0 & 0 & $\checkmark$ \\
\hline
\end{tabular}




\begin{tabular}{|c|c|c|c|c|c|}
\hline Cat. ID & Rank & $\mathcal{D}^{2}$ & Valid & $\operatorname{rk}\left(\mathcal{Z}_{2}(\mathcal{C})\right)$ & Premodular? \\
\hline \begin{tabular}{|l|}
$\mathrm{FR}_{10}^{5,0}$ \\
\end{tabular} & 5 & 8 & 1 & 2 & $\checkmark$ \\
\hline $\mathrm{FR}_{1.1}^{5,0}$ & 5 & 8 & $s$ & 5 & Symm. \\
\hline $\mathrm{FR}_{1.2}^{5,0}$ & 5 & 8 & $\checkmark$ & 2 & $\checkmark$ \\
\hline $\mathrm{FR}_{1.3}^{5,0}$ & 5 & 8 & $\checkmark$ & 5 & Symm. \\
\hline $\mathrm{FR}_{14}^{5,0}$ & 5 & 8 & $s$ & 2 & 1 \\
\hline $\mathrm{FR}_{1,5}^{5,0}$ & 5 & 8 & $s$ & 4 & $\checkmark$ \\
\hline $\mathrm{FR}_{1.6}^{5,0}$ & 5 & 8 & $s$ & 2 & $s$ \\
\hline $\mathrm{FR}_{17}^{5,0}$ & 5 & 8 & 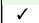 & 4 & 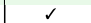 \\
\hline $\mathrm{FR}_{1,8}^{5,0}$ & 5 & 8 & $s$ & 2 & $s$ \\
\hline $\mathrm{FR}_{1.9}^{5,0}$ & 5 & 8 & $s$ & 4 & $s$ \\
\hline $\mathrm{FR}_{1: 10}^{5,0}$ & 5 & 8 & $s$ & 2 & $s$ \\
\hline $\mathrm{FR}_{1,10}^{5,0}$ & 5 & 8 & 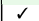 & 4 & $\checkmark$ \\
\hline $\mathrm{FR}_{1: 12}^{5,0,0}$ & 5 & 8 & $s$ & 2 & $\checkmark$ \\
\hline $\mathrm{FR}_{1.13}^{5,01}$ & 5 & 8 & $s$ & 5 & Symm. \\
\hline $\mathrm{FR}_{1.14}^{5,0}$ & 5 & 8 & 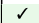 & 2 & $\checkmark$ \\
\hline $\mathrm{FR}_{1.15}^{5,0.0}$ & 5 & 8 & $\checkmark$ & 5 & Symm. \\
\hline $\mathrm{FR}_{1.16}^{5,10}$ & 5 & 8 & $s$ & 2 & $\checkmark$ \\
\hline $\mathrm{FR}_{1.17}^{5,16}$ & 5 & 8 & 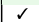 & 5 & Symm. \\
\hline $\mathrm{FR}_{1.18}^{5,0}$ & 5 & 8 & $\checkmark$ & 2 & $\checkmark$ \\
\hline $\mathrm{FR}_{1: 19}^{5,100}$ & 5 & 8 & $s$ & 2 & $s$ \\
\hline $\mathrm{FR}_{1,20}^{5,19}$ & 5 & 8 & $s$ & 4 & 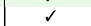 \\
\hline $\mathrm{FR}_{1: 21}^{5,00}$ & 5 & 8 & $s$ & 2 & $s$ \\
\hline $\mathrm{FR}_{1.22}^{5,01}$ & 5 & 8 & 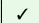 & 2 & 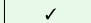 \\
\hline $\mathrm{FR}^{5,02}$ & 5 & 8 & 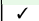 & 4 & $s$ \\
\hline $\mathrm{FR}_{1: 24}^{5,0,0}$ & 5 & 8 & $s$ & 2 & $s$ \\
\hline $\mathrm{FR}_{1.25}^{5, j, 4}$ & 5 & 8 & $s$ & 2 & $s$ \\
\hline $\mathrm{FR}_{1.26}^{5,0}$ & 5 & 8 & $s$ & 5 & Symm. \\
\hline $\mathrm{FR}_{1.27}^{5,0}$ & 5 & 8 & $s$ & 2 & $\checkmark$ \\
\hline $\mathrm{FR}_{1.28}^{5, j}$ & 5 & 8 & $s$ & 2 & $s$ \\
\hline $\mathrm{FR}_{1: 29}^{5,28}$ & 5 & 8 & $\checkmark$ & 2 & $\checkmark$ \\
\hline $\mathrm{FR}_{1,30}^{5,06}$ & 5 & 8 & $s$ & 2 & $\checkmark$ \\
\hline $\mathrm{FR}_{131}^{5,00}$ & 5 & 8 & $s$ & 2 & 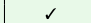 \\
\hline $\mathrm{FR}_{1 ; 32}^{5,0}$ & 5 & 8 & $\checkmark$ & 2 & $\checkmark$ \\
\hline $\mathrm{FR}_{133}^{5,0.0}$ & 5 & 8 & $s$ & 2 & $s$ \\
\hline $\mathrm{FR}_{134}^{5,03}$ & 5 & 8 & $s$ & 2 & $s$ \\
\hline $\mathrm{FR}_{1: 35}^{5,0.04}$ & 5 & 8 & $s$ & 2 & $\checkmark$ \\
\hline $\mathrm{FR}_{1: 36}^{5,0,0}$ & 5 & 8 & $s$ & 2 & $s$ \\
\hline $\mathrm{FR}_{1.37}^{5,0,0}$ & 5 & 8 & $s$ & 2 & 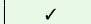 \\
\hline $\mathrm{FR}_{1 ; 38}^{5,0,}$ & 5 & 8 & $\checkmark$ & 2 & 5 \\
\hline $\mathrm{FR}_{1: 39}^{5,0}$ & 5 & 8 & $s$ & 2 & $s$ \\
\hline $\mathrm{FR}_{140}^{5,0,0}$ & 5 & 8 & $s$ & 2 & $s$ \\
\hline $\mathrm{FR}_{1: 41}^{5,00}$ & 5 & 8 & $s$ & 2 & $\checkmark$ \\
\hline $\mathrm{FR}_{1.42}^{5,01}$ & 5 & 8 & $s$ & 2 & $\checkmark$ \\
\hline $\mathrm{FR}_{143}^{5,0}$ & 5 & 8 & $s$ & 2 & $s$ \\
\hline $\mathrm{FR}_{1: 44}^{5,0}$ & 5 & 8 & $s$ & 2 & 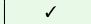 \\
\hline $\mathrm{FR}_{1.45}^{5,04}$ & 5 & 8 & $\checkmark$ & 2 & $s$ \\
\hline $\mathrm{FR}_{144}^{5,0}$ & 5 & 8 & 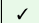 & 2 & 1 \\
\hline $\mathrm{FR}_{1 ; 47}^{5,00}$ & 5 & 8 & $s$ & 2 & $\checkmark$ \\
\hline $\mathrm{FR}_{1: 48}^{5,0,0}$ & 5 & 8 & $s$ & 2 & $s$ \\
\hline $\mathrm{FR}_{149}^{5,0}$ & 5 & 8 & $s$ & 2 & $s$ \\
\hline $\mathrm{FR}_{1 ; 50}^{\frac{1}{5,0^{3}}}$ & 5 & 8 & 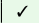 & 2 & $s$ \\
\hline $\mathrm{FR}_{1.51}^{5,0,0}$ & 5 & 8 & $s$ & 2 & $s$ \\
\hline $\mathrm{FR}_{152}^{5,0}$ & 5 & 8 & $s$ & 2 & $s$ \\
\hline $\mathrm{FR}_{1 ; 53}^{5,02}$ & 5 & 8 & $\checkmark$ & 5 & Symm. \\
\hline $\mathrm{FR}_{1.54}^{5,0}$ & 5 & 8 & $\checkmark$ & 4 & $\checkmark$ \\
\hline $\mathrm{FR}_{1,55}^{1 ; 0,0}$ & 5 & 8 & 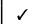 & 4 & 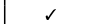 \\
\hline $\mathrm{FR}_{1 ; 56}^{5,0}$ & 5 & 8 & $s$ & 5 & Symm. \\
\hline $\mathrm{FR}_{1: 57}^{5,00}$ & 5 & 8 & $s$ & 2 & $\checkmark$ \\
\hline $\mathrm{FR}_{1,58}^{5,0,}$ & 5 & 8 & 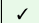 & 2 & $s$ \\
\hline $\mathrm{FR}_{1 ; 59}^{5,08}$ & 5 & 8 & $s$ & 2 & $\checkmark$ \\
\hline $\mathrm{FR}_{1: 60}^{5,0,0}$ & 5 & 8 & $s$ & 2 & $s$ \\
\hline $\mathrm{FR}_{1: 61}^{5,0}$ & 5 & 8 & $\checkmark$ & 2 & $\checkmark$ \\
\hline $\mathrm{FR}_{1 ; 62}^{5,01}$ & 5 & 8 & $\checkmark$ & 2 & $\checkmark$ \\
\hline $\mathrm{FR}_{1: 63}^{5,0,0}$ & 5 & 8 & $s$ & 2 & $\checkmark$ \\
\hline $\mathrm{FR}_{3: 0}^{5,0}$ & 5 & 12 & $s$ & 1 & Mod. \\
\hline $\mathrm{FR}_{3: 1}^{5,0}$ & 5 & 12 & $\checkmark$ & 1 & Mod. \\
\hline $\mathrm{FR}_{3.2}^{5,0}$ & 5 & 12 & $s$ & 1 & Mod. \\
\hline $\mathrm{FR}_{3.3}^{5,2}$ & 5 & 12 & $s$ & 1 & Mod. \\
\hline $\mathrm{FR}_{3: 4}^{5,0}$ & 5 & 12 & $\checkmark$ & 1 & Mod. \\
\hline $\mathrm{FR}_{3.5}^{5,0}$ & 5 & 12 & $s$ & 1 & Mod. \\
\hline $\mathrm{FR}_{3.6}^{5,0}$ & 5 & 12 & 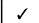 & 1 & Mod. \\
\hline $\mathrm{FR}_{3,7}^{5,0}$ & 5 & 12 & $\checkmark$ & 1 & Mod. \\
\hline $\mathrm{FR}_{4: 0}^{5,0}$ & 5 & 14 & $s$ & 5 & Symm. \\
\hline $\mathrm{FR}_{4: 1}^{5,0}$ & 5 & 14 & $\checkmark$ & 2 & $\checkmark$ \\
\hline $\mathrm{FR}_{4 ; 2}^{5,0}$ & 5 & 14 & $s$ & 2 & $\checkmark$ \\
\hline $\mathrm{FR}_{4.3}^{5,0}$ & 5 & 14 & $s$ & 2 & $s$ \\
\hline $\mathrm{FR}_{4: 4}^{5,0}$ & 5 & 14 & $\checkmark$ & 2 & $\checkmark$ \\
\hline $\mathrm{FR}_{4,5}^{5,0}$ & 5 & 14 & $s$ & 2 & $s$ \\
\hline $\mathrm{FR}_{4.6}^{5,0}$ & 5 & 14 & $s$ & 2 & $\checkmark$ \\
\hline $\mathrm{FR}_{6,0}^{5,0}$ & 5 & 24 & $s$ & 5 & Symm. \\
\hline $\mathrm{FR}_{6.1}^{5,0}$ & 5 & 24 & $s$ & 3 & $\checkmark$ \\
\hline $\mathrm{FR}_{6.2}^{5,0}$ & 5 & 24 & $\checkmark$ & 5 & Symm. \\
\hline $\mathrm{FR}_{6.3}^{5,0}$ & 5 & 24 & $\checkmark$ & 3 & $\checkmark$ \\
\hline $\mathrm{FR}_{70}^{5,0}$ & 5 & $5 \sqrt{5}+15$ & $\checkmark$ & 2 & $s$ \\
\hline $\mathrm{FR}_{71}^{5,0}$ & 5 & $5 \sqrt{5}+15$ & $s$ & 2 & $d$ \\
\hline $\mathrm{FR}_{10 \cdot 0}^{5,0}$ & 5 & $\sim 34.65$ & 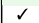 & 1 & Mod. \\
\hline $\mathrm{FR}_{10 ; 1}^{5,0}$ & 5 & $\sim 34.65$ & $\checkmark$ & 1 & Mod. \\
\hline $\mathrm{FR}_{1: 0}^{5,4}$ & 5 & 5 & $\checkmark$ & 5 & Symm. \\
\hline $\mathrm{FR}_{1.1}^{5,4}$ & 5 & 5 & $\checkmark$ & 1 & Mod. \\
\hline $\mathrm{FR}_{1: 2}^{5,4}$ & 5 & 5 & $\checkmark$ & 1 & Mod. \\
\hline $\mathrm{FR}_{1.3}^{5,4}$ & 5 & 5 & $\checkmark$ & 1 & Mod. \\
\hline $\mathrm{FR}_{1: 4}^{5,4}$ & 5 & 5 & $\checkmark$ & 1 & Mod. \\
\hline
\end{tabular}




\section{References}

1. Haldane, F.D.M., Rezayi, E.H.: Periodic Laughlin-Jastrow wave functions for the fractional quantized Hall effect. Phys. Rev. B 31, 2529 (1985)

2. Wen, X.G.: Vacuum degeneracy of chiral spin states in compactified space. Phys. Rev. B 40, 7387 (1989)

3. Wen, X.G., Niu, Q.: Ground-state degeneracy of the fractional quantum Hall states in the presence of a random potential and on high-genus Riemann surfaces. Phys. Rev. B 41, 9377 (1990)

4. Wen, X.-G.: Quantum field theory of many-body systems: from the origin of sound to an origin of light and electrons. Oxford University Pres, Oxford (2004)

5. Kitaev, A.Y.: Fault-tolerant quantum computation by anyons. Ann. Phys. 303, 2 (2003). arXiv:quant-ph/9707021

6. Brown, B.J., Loss, D., Pachos, J.K., Self, C.N., Wootton, J.R.: Quantum memories at finite temperature. Rev. Mod. Phys. 88, 045005 (2016). arXiv:1411.6643

7. Wilczek, F.: Magnetic flux, angular momentum, and statistics. Phys. Rev. Lett. 48, 1144 (1982a)

8. Wilczek, F.: Quantum mechanics of fractional-spin particles. Phys. Rev. Lett. 49, 957 (1982b)

9. Levin, M.A., Wen, X.-G.: String-net condensation: A physical mechanism for topological phases. Phys. Rev. B 71, 045110 (2005). arXiv:cond-mat/0404617

10. Walker, K., Wang, Z.: (3+1)-TQFTs and topological insulators. Front. Phys. 7, 150 (2012). arXiv:1104.2632

11. Hu, Y., Luo, Z.-X., Pankovich, R., Wan, Y., Wu, Y.-S.: Boundary Hamiltonian theory for gapped topological phases on an open surface. J. High Energy Phys. 2018, 134 (2018). arXiv:1706.03329

12. Hu, Y., Wan, Y., Wu, Y.-S.: Boundary Hamiltonian theory for gapped topological orders. Chin. Phys. Lett. 34, 077103 (2017). arXiv: 1706.00650

13. Chen, X., Gu, Z.-C., Wen, X.-G.: Local unitary transformation, long-range quantum entanglement, wave function renormalization, and topological order. Phys. Rev. B 82, 155138 (2010). arXiv:1004.3835

14. Wang, Z., Chen, X.: Twisted gauge theories in three-dimensional Walker-Wang models. Phys. Rev. B 95, 115142 (2017). arXiv:1611.09334

15. Hamma, A., Ionicioiu, R., Zanardi, P.: Bipartite entanglement and entropic boundary law in lattice spin systems. Phys. Rev. A 71, 022315 (2005a). arXiv:quant-ph/0409073

16. Kitaev, A., Preskill, J.: Topological entanglement entropy. Phys. Rev. Lett. 96, 110404 (2006). arXiv:hep-th/0510092

17. Levin, M., Wen, X.-G.: Detecting topological order in a ground state wave function. Phys. Rev. Lett. 96, 110405 (2006). arXiv:cond-mat/0510613

18. Eisert, J., Cramer, M., Plenio, M.B.: Colloquium: Area laws for the entanglement entropy. Rev. Mod. Phys. 82, 277 (2010). arXiv:0808.3773

19. Dong, S., Fradkin, E., Leigh, R.G., Nowling, S.: Topological entanglement entropy in Chern-Simons theories and quantum Hall fluids. J. High Energy Phys. 2008, 016 (2008). arXiv:0802.3231

20. Brown, B.J., Bartlett, S.D., Doherty, A.C., Barrett, S.D.: Topological entanglement entropy with a twist. Phys. Rev. Lett. 111, 220402 (2013). arXiv:1303.4455

21. Bonderson, P., Knapp, C., Patel, K.: Anyonic entanglement and topological entanglement entropy. Ann. Phys. 385, 399 (2017). arXiv:1706.09420

22. Shi, B., Kato, K., Kim, I.H.: Fusion rules from entanglement. Ann. Phys. 418, 168164 (2020). arXiv: 1906.09376

23. Zhang, Y., Grover, T., Turner, A., Oshikawa, M., Vishwanath, A.: Quasiparticle statistics and braiding from ground-state entanglement. Phys. Rev. B 85, 235151 (2012). arXiv:1111.2342

24. Castelnovo, C., Chamon, C.: Topological order in a three-dimensional toric code at finite temperature. Phys. Rev. B 78, 155120 (2008). arXiv:0804.3591

25. Grover, T., Turner, A.M., Vishwanath, A.: Entanglement entropy of gapped phases and topological order in three dimensions. Phys. Rev. B 84, 195120 (2011). arXiv:1108.4038

26. Hamma, A., Zanardi, P., Wen, X.-G.: String and membrane condensation on three-dimensional lattices. Phys. Rev. B 72, 035307 (2005b). arXiv:cond-mat/0411752

27. von Keyserlingk, C.W., Burnell, F.J., Simon, S.H.: Three-dimensional topological lattice models with surface anyons. Phys. Rev. B 87, 045107 (2013). arXiv:1208.5128

28. Bullivant, A., Pachos, J.K.: Entropic manifestations of topological order in three dimensions. Phys. Rev. B 93, 125111 (2016). arXiv: 1504.02868

29. Kim, I.H., Brown, B.J.: Ground-state entanglement constrains low-energy excitations. Phys. Rev. B 92, 115139 (2015). arXiv:1410.7411

30. Shi, B., Lu, Y.-M.: Characterizing topological order by the information convex. Phys. Rev. B 99, 035112 (2019). arXiv:1801.01519

31. Etingof, P., Gelaki, S., Nikshych, D., Ostrik, V.: Tensor categories, Mathematical Surveys and Monographs, vol. 205, p. pp. xvi+343. American Mathematical Society, Providence, RI (2015) 
32. Kitaev, A.: Anyons in an exactly solved model and beyond. Ann. Phys. 321, 2 (2006). arXiv:cond-mat/0506438

33. Bonderson, P.H.: Non-Abelian Anyons and Interferometry, Ph.D. thesis, California Institute of Technology (2007)

34. Beer, K., Bondarenko, D., Hahn, A., Kalabakov, M., Knust, N., Niermann, L., Osborne, T.J., Schridde, C., Seckmeyer, S., Stiegemann, D.E., Wolf, R.: From categories to anyons: a travelogue, arXiv:1811.06670 (2018)

35. We refrain from drawing arrows on the diagrams, instead using the convention that all lines are oriented upwards

36. Etingof, P., Nikshych, D., Ostrik, V.: On fusion categories. Ann. Math. 162, 581 (2005). arXiv:math/0203060

37. Galindo, C.: On braided and ribbon unitary fusion categories. Can. Math. Bull. 57, 506 (2014). arXiv:1209.2022

38. Müger, M.: On the structure of modular categories. Proc. Lond. Math. Soc. 87, 291 (2003a). arXiv:math/0201017

39. Bakalov, B., Kirillov, A.A.: Lectures on Tensor Categories and Modular Functors, vol. 21. American Mathematical Society, Providence (2001)

40. Bonderson, P., Delaney, C., Galindo, C., Rowell, E.C., Tran, A., Wang, Z.: On invariants of modular categories beyond modular data. J. Pure Appl. Algebra 223, 4065 (2019). arXiv:1805.05736

41. Müger, M.: From Subfactors to Categories and Topology I. Frobenius algebras in and Morita equivalence of tensor categories. J. Pure Appl. Algebra 180, 81 (2003b). arXiv:math/0111204

42. Bridgeman, J.C.: UnitaryPremodularCategoryData, github.com/JCBridgeman/UnitaryPremodular CategoryData, Github repository (2020)

43. The lattice is the dual of the triangulation

44. Williamson, D.J., Wang, Z.: Hamiltonian models for topological phases of matter in three spatial dimensions. Ann. Phys. 377, 311 (2017). arXiv:1606.07144

45. Crane, L., Yetter, D.: A categorical construction of 4D topological quantum field theories. Quant. Topol. 3, 120 (1993). arXiv:hep-th/9301062

46. Crane, L., Kauffman, L.H., Yetter, D.N.: State-sum invariants of 4-manifolds. J. Knot Theory Ramif. 6, 177 (1997). arXiv:hep-th/9409167

47. The global vector space can be made a tensor product space by, for example, choosing the dimension of the vertex space to be the largest of all the fusion spaces

48. Wang, Z.: Topological Phases of Matter: Exactly Solvable Models and Classification, Ph.D. thesis, California Institute of Technology (2019)

49. Fuchs, J., Runkel, I., Schweigert, C.: TFT construction of RCFT correlators I: partition functions. Nucl. Phys. B 646, 353 (2002). arXiv:hep-th/0204148

50. Kitaev, A.Y., Kong, L.: Models for gapped boundaries and domain walls. Commun. Math. Phys. 313, 351 (2012). arXiv:1104.5047

51. Fuchs, J., Schweigert, C., Valentino, A.: Bicategories for boundary conditions and for surface defects in 3-D TFT. Commun. Math. Phys. 321, 543 (2013). arXiv:1203.4568

52. Fuchs, J., Priel, J., Schweigert, C., Valentino, A.: On the Brauer groups of symmetries of abelian DijkgraafWitten theories. Commun. Math. Phys. 339, 385 (2015). arXiv:1404.6646

53. Private communications with David Aasen

54. Bravyi, S.B., Kitaev, A.Y.: Quantum codes on a lattice with boundary, arXiv:quant-ph/9811052 (1998)

55. von Keyserlingk, C.W., Burnell, F.J.: Walker-Wang models and axion electrodynamics. Phys. Rev. B 91, 045134 (2015). arXiv: 1405.2988

56. Bravyi, S.: Unpublished

57. Cano, J., Hughes, T.L., Mulligan, M.: Interactions along an entanglement cut in $2+1 D$ Abelian topological phases. Phys. Rev. B 92, 075104 (2015). arXiv: 1411.5369

58. Zou, L., Haah, J.: Spurious long-range entanglement and replica correlation length. Phys. Rev. B 94, 075151 (2016). arXiv:1604.06101

59. Williamson, D.J., Dua, A., Cheng, M.: Spurious topological entanglement entropy from subsystem symmetries. Phys. Rev. Lett. 122, 140506 (2019). arXiv:1808.05221

60. Kato, K., Brandão, F.G.: Toy model of boundary states with spurious topological entanglement entropy, Phys. Rev. Research 2, 032005(R), arXiv:1911.09819 (2020)

61. Levin, M.: String-net condensation and topological phases in quantum spin systems, Ph.D. thesis, Massachusetts Institute of Technology (2006)

62. Tambara, D., Yamagami, S.: Tensor categories with fusion rules of self-duality for finite abelian groups. J. Algebra 209, 692 (1998)

63. Siehler, J.A.: Braided near-group categories, arXiv:math/0011037 (2000)

64. Joyal, A., Street, R.: Braided tensor categories. Adv. Math. 102, 20 (1993) 
65. Ostrik, V.: Module categories over the Drinfeld double of a finite group. Int. Math. Res. Not. 2003, 1507 (2003). arXiv:math/0202130

66. Bullivant, A., Delcamp, C.: Gapped boundaries and string-like excitations in $(3+1) d$ gauge models of topological phases. J. High Energy Phys. 2021, 25 (2021). arXiv:2006.06536

67. Bruillard, P.: Rank 4 premodular categories. New York J. Math. 22, 775 (2016). arXiv:1204.4836

68. Preskill, J.: Lecture notes for physics 219: Quantum computation, chapter 9 (2004)

69. Ostrik, V.: Fusion categories of rank 2. Math. Res. Lett. 10, 177 (2003b). arXiv:math/0203255

70. Ostrik, V.: Pre-modular categories of rank 3. Mosc. Math. J. 8, 111 (2008). arXiv:math/0503564

71. Rowell, E., Stong, R., Wang, Z.: On classification of modular tensor categories. Commun. Math. Phys. 292, 343 (2009). arXiv:0712.1377

72. Bruillard, P., Ortiz-Marrero, C.M.: Classification of rank 5 premodular categories. J. Math. Phys. 59, 011702 (2018). arXiv: 1612.08769

73. Yu, Z.: On slightly degenerate fusion categories. J. Algebra 559, 408 (2020). arXiv:1903.06345

74. List of small multiplicity-free fusion rings. http://www.thphys.nuim.ie/AnyonWiki/index.php/List_of_ small_multiplicity-free_fusion_rings. Accessed 1 Nov (2020)

Communicated by C. Schweigert 